\title{
The Role of the Magnetic Field in the Interstellar Medium of the Post-Starburst Dwarf Irregular Galaxy NGC 1569
}

\author{
Amanda A. Kepley \\ Department of Astronomy, University of Wisconsin-Madison, 475 North Charter Street, \\ Madison, WI 53706 \\ kepley@astro.wisc.edu \\ Stefanie Mühle \\ Joint Institute for VLBI in Europe, Postbus 2, 7990 AA Dwingeloo, The Netherlands \\ muehle@jive.nl \\ John Everett \\ Department of Astronomy, University of Wisconsin-Madison, 475 North Charter Street, \\ Madison, WI 53706 \\ everett@physics.wisc.edu \\ Ellen G. Zweibel \\ Department of Astronomy, University of Wisconsin-Madison, 475 North Charter Street, \\ Madison, WI 53706 \\ zweibel@astro.wisc.edu \\ Eric M. Wilcots \\ Department of Astronomy, University of Wisconsin-Madison, 475 North Charter Street, \\ Madison, WI 53706 \\ ewilcots@astro.wisc.edu \\ Uli Klein \\ Argelander-Institut für Astronomie, Universität Bonn, Auf dem Hügel 71, D-53121 Bonn, \\ Germany \\ uklein@astro.uni-bonn.de
}

ABSTRACT 
NGC 1569 is a nearby dwarf irregular galaxy which underwent an intense burst of star formation 10 to 40 Myr ago. We present observations that reach surface brightnesses two to eighty times fainter than previous radio continuum observations and the first radio continuum polarization observations of this galaxy at $20 \mathrm{~cm}, 13 \mathrm{~cm}, 6 \mathrm{~cm}$, and $3 \mathrm{~cm}$. These observations allow us to probe the relationship of the magnetic field of NGC 1569 to the rest of its interstellar medium. We confirm the presence of an extended radio continuum halo at $20 \mathrm{~cm}$ and see for the first time the radio continuum feature associated with the western $\mathrm{H} \alpha \mathrm{arm}$ at wavelengths shorter than $20 \mathrm{~cm}$. Although, in general, the spectral indices derived for this galaxy steepen as one moves into the halo of the galaxy, there are filamentary regions of flat spectral indices extending to the edge of the galaxy. The spectral index trends in this galaxy support the theory that there is a convective wind at work in this galaxy. There is strong polarized emission at $3 \mathrm{~cm}$ and $6 \mathrm{~cm}$ and weak polarized emission at $20 \mathrm{~cm}$ and $13 \mathrm{~cm}$. We estimate that the thermal fraction is $40-50 \%$ in the center of the galaxy and falls off rapidly with height above the disk. Using this estimate, we derive a total magnetic field strength of $38 \mu \mathrm{G}$ in the central regions and $10-15 \mu \mathrm{G}$ in the halo. The magnetic field is largely random in the center of the galaxy; the uniform field is $\sim 3-9 \mu \mathrm{G}$ and is strongest in the halo. Using our total magnetic field strength estimates and the results of previous observations of NGC 1569, we find that the magnetic pressure is the same order of magnitude but, in general, a factor of a few less than the other components of the interstellar medium in this galaxy. The uniform magnetic field in NGC 1569 is closely associated with the $\mathrm{H} \alpha$ bubbles and filaments. We suggest that a supernova-driven dynamo may be operating in this galaxy. Based on our pressure estimates and the morphology of the magnetic field, the outflow of hot gas from NGC 1569 is clearly shaping the magnetic field, but the magnetic field in turn may be aiding the outflow by channeling gas out of the disk of the galaxy. Dwarf galaxies with extended radio continuum halos like that of NGC 1569 may play an important role in magnetizing the intergalactic medium.

Subject headings: galaxies: individual (NGC 1569) — galaxies: ISM — galaxies: irregular - galaxies: magnetic fields - galaxies: starburst — radio continuum: galaxies 


\section{Introduction}

The structure of a galaxy's interstellar medium (ISM) is shaped by its stars. Stars dissociate and ionize the surrounding cocoon of cold gas from which they formed. Winds from massive stars and supernovae explosions also create bubbles of hot gas. The combined effects of multiple stars in a cluster create larger bubbles of hot gas referred to superbubbles. The low mass of dwarf irregular galaxies makes their ISM particularly vulnerable to disruption by intense episodes of star formation (starbursts). Starbursts inject turbulence into the interstellar medium and may provide enough energy to drive an outflow of gas from the galaxy (Mac Low \& Ferrara 1999; de Avillez \& Breitschwerdt 2005; Tenorio-Tagle et al. 2006; Cooper et al. 2008). While there have been several studies on the effects of starbursts on the warm (Martin 1998), hot (Ott et al. 2005b), and cold gas (Schwartz \& Martin 2004) in dwarf irregular galaxies, little attention has been paid to the role of magnetic fields in starbursting dwarf irregular galaxies.

The presence of magnetic fields can influence the behavior of the ISM in these galaxies in several ways. First, the field is an important source of pressure for the interstellar medium. Based on observations of the ISM of the Milky Way (for a comprehensive review see Ferrière 2001), one often assumes that the interstellar medium of galaxies is in equipartition with approximately equal energy in the magnetic field, cosmic rays, and turbulence. Second, given the shallow gravitational potential well of these galaxies, the magnetic field may be strong enough to be dynamically important and aid in expelling gas from the disk of the galaxy. The configuration of the field, however, may help or hinder the outflow. Although earlier work by Tomisaka (1990) and Mineshige et al. (1993) came to the conclusion that magnetic fields parallel to the disk could prevent the breakout of superbubbles from the disk, more recent 3D simulations by de Avillez \& Breitschwerdt (2005) show that while a disk magnetic field may delay the break out of superbubbles, the amount of hot gas vented from superbubbles is not greatly affected by the addition of a magnetic field. For the case of a magnetic field perpendicular to the galactic disk, Breitschwerdt et al. (1991, 1993) present models of a cosmic-ray driven wind, where the magnetic field transfers momentum and energy from cosmic rays to thermal gas thus helping to drive the wind. Everett et al. (2008, 2009) later extended these models to explain the distribution of soft Galactic X-rays and $408 \mathrm{MHz}$ emission towards the Galactic Center. The cosmic rays, acting through the magnetic field, may increase mass loss rates or increase the terminal velocity of the wind depending on the strength and geometry of the magnetic field (Everett et al. 2008, 2009).

There are a handful of starburst galaxies with detailed magnetic field observations including M82 (Klein et al. 1988; Reuter et al. 1994), NGC 253 (Carilli et al. 1992; Beck et al. 1994: Heesen et al. 2009), NGC 4666 (Dahlem et al. 1997), NGC 1808 (Dahlem et al. 1990), 
and NGC 4569 (Chyży et al. 2006). The common features in all these objects are extended radio continuum emission well beyond the disk of the galaxy and spurs of polarized radio continuum emission extending from the disk of the galaxy to the halo of the galaxy with their derived magnetic field vectors roughly perpendicular to the disk. However, all these galaxies are quite massive - NGC 253, NGC 4666, NGC 1808, and NGC 4569 have dynamical masses on the order of $10^{11} \mathrm{M}_{\odot}$, while M82 has a dynamical mass on the order of $10^{10} \mathrm{M}_{\odot}$. A low-mass galaxy like a dwarf irregular, which has a dynamical mass on the order of $10^{9} \mathrm{M}_{\odot}$, provides a more rigorous test of the role of the magnetic field in a starburst.

One of the most extreme starbursts in the local universe is the dwarf irregular galaxy NGC 1569. This galaxy, which is only 3.36 Mpc away (Grocholski et al. 2008), underwent an intense burst of star formation (star formation rate $\sim 1-3 M_{\odot} \mathrm{yr}^{-1} \mathrm{kpc}^{-2}$ ) in the recent past ( 10-40 Myr ago) (Vallenari \& Bomans 1996; Greggio et al. 1998; Aloisi et al. 2001; Angeretti et al. 2005). Today, NGC 1569 is in a post-starburst phase, although it is still vigorously forming stars (Greggio et al. 1998). The subsequent evolution and demise of the high-mass stars formed in the starburst has injected an enormous amount of energy into the interstellar medium of NGC 1569 causing massive disruption of the gas. The H $\alpha$ structure of this highly inclined galaxy $(i=63$; Stil \& Israel 2002) consists of filaments of emission extending well into the halo of the galaxy. The most prominent $\mathrm{H} \alpha$ filament is referred to as the "western arm" (note that this is not a spiral arm, but the limb of an $\mathrm{H} \alpha$ bubble). Investigations of the $\mathrm{H} \alpha$ kinematics (Tomita et al. 1994; Heckman et al. 1995; Martin 1998; Westmoquette et al. 2007a, b, 2008) reveal that the central regions of the galaxy are dominated by turbulent motions, while the outer regions show clear signs of a large-scale outflow of ionized gas. The neutral hydrogen velocity field is completely disrupted in the central regions of NGC 1569, but rotates as a solid-body in the outer regions (Mühle et al. 2005). X-ray observations (Heckman et al. 1995; della Ceca et al. 1996; Martin et al. 2002; Ott et al. 2005a b have revealed the widespread presence of hot, partially shock-heated, gas bounded by the $\mathrm{H} \alpha$ filaments throughout the galaxy. This gas has a high $\alpha$-to-Fe ratio indicating that the hot gas is enriched with the products of type II supernovae (Martin et al. 2002). Although NGC 1569 clearly has gas being expelled from its central regions and the gas likely has enough energy to escape (Martin 1998; Ott et al. 2005b), how much material, if any, will actually escape the galactic potential remains controversial. A detailed study of the magnetic field strength and structure of NGC 1569 is a crucial component for understanding the outflow of gas from this galaxy.

The magnetic field of a galaxy can be traced using its radio continuum emission, which is a combination of synchrotron radiation generated by relativistic electrons spiraling along magnetic field lines and free-free emission generated by thermal electrons. The resolved radio continuum emission from NGC 1569 has been observed by Seaquist \& Bignell $(1976)$, Condon 
(1983), Klein \& Gräve (1986) (1.2 cm [24.5 GHz] data only), Israel \& de Bruyn (1988), and Lisenfeld et al. (2004). Seaquist \& Bignell (1976) found that most of the regions they detected in NGC 1569 are thermal in nature; however, these observations were only sensitive to a limited range of spatial scales with little sensitivity to large-scale emission. Condon (1983) presented VLA observations of NGC 1569 showing an extended radio continuum halo at $20 \mathrm{~cm}(1.4 \mathrm{GHz})$, but did not discuss them extensively. The Effelsberg 100-m observations of Klein \& Gräve (1986) only resolved NGC 1569 at $1.2 \mathrm{~cm}(24.5 \mathrm{GHz})$. At this wavelength, they detected faint emission associated with the western arm for the first time at a frequency higher than $20 \mathrm{~cm}(1.4 \mathrm{GHz})$. Israel \& de Bruyn (1988) presented observations of the radio continuum emission from NGC 1569 at $50 \mathrm{~cm}, 20 \mathrm{~cm}$, and $6 \mathrm{~cm}(0.6 \mathrm{GHz}, 1.4 \mathrm{GHz}$, and $5.0 \mathrm{GHz}$, respectively) and discuss in detail the faint extended radio halo around this galaxy first found by Condon (1983) and visible in their observations at both $50 \mathrm{~cm}$ and $20 \mathrm{~cm}$. They identified a break in the non-thermal radio continuum spectrum of this galaxy at $3.75 \mathrm{~cm}$ $(8 \pm 1 \mathrm{GHz})$. Lisenfeld et al. (2004) modeled the integrated radio spectrum (including the break) and concluded that the radio continuum spectrum is likely the result of cosmic-ray escape in a convective wind, which fits with the picture of NGC 1569 as a galaxy undergoing a tremendous outflow of material.

In this paper, we present multi-wavelength radio continuum polarization observations of the post-starburst dwarf irregular galaxy NGC 1569, which will allow us to determine the strength of the magnetic field in this galaxy and trace its structure. This data set represents the most sensitive set of radio continuum observations of NGC 1569 to date and the first observations of its polarized emission at $20 \mathrm{~cm}, 13 \mathrm{~cm}, 6 \mathrm{~cm}$, and $3 \mathrm{~cm}$. We describe our data set in $\S 2$ and present the basic properties of the total intensity and polarized continuum emission of NGC 1569 in $\S 3$. In $\S 4$, we investigate the properties of the magnetic field of NGC 1569. We place our observations in the context of what is known about the interstellar medium of NGC 1569 in $\S 5$. Finally, in $\S$ 6, we present a summary of our work and our conclusions.

\section{Observations and Data Reduction}

We observed the radio continuum emission from NGC 1569 at 20, 13, 6, and $3 \mathrm{~cm}$. The 20 and $13 \mathrm{~cm}$ observations were taken with the Westerbork Synthesis Radio Telescope (WSRT) ${ }^{1}$. The WSRT is an east-west interferometer consisting of fourteen 25-m antennas.

\footnotetext{
1 The Westerbork Synthesis Radio Telescope is operated by ASTRON (Netherlands Foundation for Research in Astronomy) with support from the Netherlands Foundation for Scientific Research (NWO).
} 
Ten of the antennas are at fixed positions, while the other four antennas may be moved along an east-west track. At 6 and $3 \mathrm{~cm}$, we used both the Very Large Array (VLA) ${ }^{2}$, which is an interferometer with twenty-seven 25-m antennas arranged in a Y-shape, and the Effelsberg 100-m Radio Telescop ${ }^{3}$, which is a single-dish telescope. The WSRT and VLA observations provide us with the spatial resolution necessary to detect small-scale magnetic field structure that would be depolarized by the large beam of the Effelsberg observations. The Effelsberg observations are necessary to recover the emission on the largest spatial scales, which may be resolved out by the VLA at 6 and $3 \mathrm{~cm}$. At 20 and $13 \mathrm{~cm}$, the largest angular scales imaged by the WSRT are approximately $19^{\prime}$ and $17^{\prime}$, which are both much greater than the size of the galaxy, so there is very little chance of large-scale emission from NGC 1569 being resolved out at these wavelengths. Below we discuss the calibration and imaging of the data in detail.

\section{1. $20 \mathrm{~cm}$ Data}

The $20 \mathrm{~cm}$ data were taken with the WSRT as part of project R01A/12 (PI: S. Mühle) on 5-6 and 6-7 November 2000. The project also included observations of the radio continuum of NGC 1569 at $49 \mathrm{~cm}$, but the $49 \mathrm{~cm}$ data were unusable due to extensive radio frequency interference. To calibrate the instrumental polarization, each observing session included an observation of a source with a known polarization angle and an unpolarized source. These sources were also used to calibrate the flux density scale. The time on-source for each day was 12 hours. To maximize the coverage of the $u-v$ plane, we used two different array configurations: the $72 \mathrm{~m}$ configuration and the $36 \mathrm{~m}$ configuration. The DCB backend was configured to produce eight $10 \mathrm{MHz}$-wide intermediate frequency (IF) channels each with full polarization products.

Table 1 summarizes the $20 \mathrm{~cm}$ observations. The data were calibrated and imaged using the NEWSTAR package (Brouw 1996), following standard calibration procedures and using a hybrid cleaning method, which includes self-calibration of the gains and phases of the Stokes I, U, and V data. In Stokes Q, only the phases were self-calibrated, since a self-calibration of the gains would force Stokes $Q$ equal to zero in the case of linear feeds $(\mathrm{Q}=\mathrm{XX}-\mathrm{YY})$. Table 2 gives details for the final images. The upper left panel of Figure 1 shows the total intensity

\footnotetext{
${ }^{2}$ The VLA is part of the National Radio Astronomy Observatory. The National Radio Astronomy Observatory is a facility of the National Science Foundation operated under cooperative agreement by Associated Universities, Inc.

3 Operated by MPIfR (Max-Planck-Institut für Radioastronomie).
} 
map of the $20 \mathrm{~cm}$ data. The final image includes low-level $\left(20 \mu \mathrm{Jy}_{\mathrm{beam}}{ }^{-1}\right)$ ripples in the total intensity that are artifacts of the imaging process for this data.

\section{2. $\quad 13 \mathrm{~cm}$ Data}

The $13 \mathrm{~cm}$ data were obtained with the WSRT (project ID: R03A/25, PI: S. Mühle) on 4-5 December 2002 and 18-19 January 2003. The time on-source for each observing session was 12 hours. Observations of a polarized source and an unpolarized source were taken before and after each session to calibrate the instrumental polarization as well as the bandpasses and the flux density scale. A summary of the WSRT $13 \mathrm{~cm}$ observations used in this paper and their calibration is given in Table 3. The data were taken in the 36-m and 72-m configurations and DZB backend was configured to produce eight $20 \mathrm{MHz}$-wide IFs, each with full polarization products and 64 channels.

Since the $13 \mathrm{~cm}$ receivers on the WSRT have circular feeds, we calibrated the data using the standard calibration routines in AIPS, which, in general, assume circularly polarized feeds. Reducing WSRT data in AIPS has two important differences compared to reducing typical VLA observations. First, the WSRT is a redundantly spaced array, so observing phase calibrators periodically during the observing session is not necessary because the redundant baselines provide multiple measurements of the same visibility. Self-calibration is used to solve for the variation of phase as a function of time. The second complication is that the WSRT dishes are equatorially mounted, so the parallactic angle does not change as a function of time. Therefore, a single observation of an unpolarized source must be used to calibrate the instrumental polarization. This is in contrast to the VLA where the polarization response of the antennas is usually calibrated using a weakly polarized source observed over a large range in parallactic angle. Practically, it means that the task LPCAL must be used to calibrate the instrumental polarization instead of the task PCAL.

We used the AIPS task CALIB to self-calibrate the data using models of the galaxy generated by imaging and cleaning the data. We did several iterations of phase-only selfcalibration with solution intervals of between 5 and 10 minutes and restricted the solutions to those baselines where the signal-to-noise ratio was greater than 3. After the phases had been improved, we did a final round of amplitude and phase self-calibration with our best model of the source, which included as much of the diffuse flux as possible. The solution interval was between 20 and 30 minutes and only baselines where the signal-to-noise ratio was greater than 5 were included in the solution. Each observation period was self-calibrated separately and the individual periods combined together. Finally, the combined data was amplitude and phase self-calibrated with a solution interval of 12 hours including only those 
baselines with signal-to-noise ratio greater than 5 to ensure a uniform amplitude scale.

We produced two final images: a robust $=0$ image and an image matched to the resolution of the high-resolution $20 \mathrm{~cm}$ image $\left(\sim 13^{\prime \prime}\right)$. The robust $=0$ image has a weighting between uniform and natural and provides the best compromise between the lower noise given by natural weighting and the minimization of the sidelobes by uniform weighting. Table 2 lists the details of the final images. The upper right panel of Figure 1 shows the robust=0 image.

\section{3. $\quad 6 \mathrm{~cm} \mathrm{\&} 3 \mathrm{~cm}$ Data}

The interferometer data at these wavelengths were taken with the VLA under program IDs AM643 (D array observations) and AM694 (C array observations). The PI for both programs was S. Mühle. A full set of polarization products (RR, RL, LR, LL) were produced for each of 2 IFs with a bandwidth per IF of $50 \mathrm{MHz}$. The total time on-source was 3.97 hours for the $6 \mathrm{~cm}(4.8 \mathrm{GHz})$ D-array observations, 14.7 hours for the $6 \mathrm{~cm}(4.8 \mathrm{GHz}) \mathrm{C}$-array observations, and 10.5 hours for the $3 \mathrm{~cm}(8.4 \mathrm{GHz})$ D-array observations. We lost 2 hours of time on source during the $3 \mathrm{~cm}$ observations on 23 September 2000 due to high winds. We calibrated the data in AIPS following the standard calibration procedures detailed in the AIPS cookbook (Greisen 2006). We self-calibrated the data in AIPS using the same procedure as for the $13 \mathrm{~cm}$ data (see $\S 2.2$. Table 4 summarizes our observations and calibration parameters.

Whenever possible, we used $1331+305$ (3C286) as our absolute polarization angle calibrator, since the polarization angles of all other calibrators are derived from this source. If $1331+305$ was not available, $0521+166$ was used as an absolute polarization angle calibrator. The polarization angle of $0521+166$ in the last row of Table 4 was determined using observing sessions where we observed both $0521+166$ and $1331+305$. The absolute polarization angle for $0521+166$ on 9 August was determined from observations on 10 August, on 11 and 17 August from observations on 19 September, on 23 September from observations on that day. Our observations on 19 September indicate that the absolute polarization angle of $0521+166$ varied by $\pm 2^{\circ}$ over the entire observing run.

To correct the $6 \mathrm{~cm}$ and $3 \mathrm{~cm}$ VLA data for the effects of missing short spacing: 4 , we obtained $6 \mathrm{~cm}(4.85 \mathrm{GHz})$ and $3 \mathrm{~cm}(10.45 \mathrm{GHz})$ data with the Effelsberg $100-\mathrm{m}$ radio telescope in December 2000 and January 2001, respectively. The data at both wavelengths were

\footnotetext{
${ }^{4}$ It is not necessary to correct the $20 \mathrm{~cm}$ and $13 \mathrm{~cm}$ data for the effect of missing short spacings since the largest angular scale imaged by the WSRT at these frequencies is much larger than the size of the galaxy.
} 
reduced and calibrated using the NOD2 data reduction package (Haslam 1974). The $6 \mathrm{~cm}$ $(4.85 \mathrm{GHz})$ Effelsberg receiver has two feeds, which allows one to eliminate atmospheric noise using the dual-beam technique described in Emerson et al. (1979). The telescope scans in azimuth to align the two feeds. To remove raster noise, we averaged together individual maps taken across a range of parallactic angles. Atmospheric noise at $3 \mathrm{~cm}(10.45 \mathrm{GHz})$ was minimized by using the software beam-switching technique on the data from the four feeds of this receiver. The residual scanning noise in the individual images was reduced by down-weighting the regions in Fourier space where raster noise is present (Emerson \& Gräve 1988). The individual maps were then averaged to produce the final maps. Table 5 gives a synopsis of our Effelsberg observations.

Since the VLA and Effelsberg $3 \mathrm{~cm}$ images were taken at two different frequencies (8.46 $\mathrm{GHz}$ and $10.45 \mathrm{GHz}$ ), we scaled the Effelsberg Stokes I image by the spectral index determined by Lisenfeld et al. (2004): $\alpha=-0.47$. We did not scale the Effelsberg Stokes Q and U images because we do not know how NGC 1569's polarized emission changes between 8.46 $\mathrm{GHz}$ and $10.45 \mathrm{GHz}$. We can, however, constrain the rotation measure between these two frequencies. For a change of $10^{\circ}$ in the angle of the polarization vector, there would have to be a rotation measure of at least $400 \mathrm{rad} \mathrm{m}^{-2}$. In $\S 4.2$, we determine the rotation measures for NGC 1569 between $6 \mathrm{~cm}$ and $3 \mathrm{~cm}$. Most of the measured values are less than $400 \mathrm{rad} \mathrm{m}^{-2}$ (see Figure 10), so not scaling the flux in the Effelsberg 10.45 GHz Stokes Q and U images should not be problematic. However, rotation measures greater than, or comparable, to 400 rad $\mathrm{m}^{-2}$ may be affected by combining unscaled Stokes Q and U data taken at two different frequencies.

To combine the interferometric and single-dish data, we used the immerge task in Miriad, which combines the images in the spatial frequency domain. In this approach, the different calibration scales of the Effelsberg and VLA data are dealt with by scaling the low-resolution visibilities so that they match the high-resolution visibilities in the region of the $u-v$ plane where the observations overlap ( 0.4 to $1.6 \mathrm{k} \lambda$ for the $6 \mathrm{~cm}$ data and 0.725 to $2.8 \mathrm{k} \lambda$ for the $3 \mathrm{~cm}$ data). The scaling factors for the visibilities in the overlap region as determined by immerge were 0.8196 ( $6 \mathrm{~cm}$, robust $=0$ image $), 0.856\left(6 \mathrm{~cm}, 13^{\prime \prime}\right.$ image $), 0.9263(3 \mathrm{~cm}$, robust $=0$ image $)$, and $0.9330\left(3 \mathrm{~cm}, 13^{\prime \prime}\right.$ image $)$. We used the scaling factors determined by the Stokes I images for the Stokes Q and U images.

Details on the final $6 \mathrm{~cm}$ and $3 \mathrm{~cm}$ images are given in Table 2. Figures 1 and 2 show the robust $=0$ weighted $6 \mathrm{~cm}$ and $3 \mathrm{~cm}$ images. The total fluxes of the final images are within $10 \%$ of the total fluxes measured with Effelsberg (see Figure 3). 


\section{Properties of the Radio Continuum Emission}

The total intensity of the radio continuum emission from a source gives one information about the combined free-free and synchrotron emission in that source. By separating the synchrotron emission from the free-free emission, one can estimate the total magnetic field strength (see $\$ \$ 4.1$ and 4.2 . Since free-free emission is unpolarized and synchrotron emission is polarized, any polarized emission is inherently synchrotron emission and traces of the uniform component of the magnetic field (see $\S 4.3$ ).

\subsection{Total Intensity}

Previous resolved studies of NGC 1569 at radio continuum wavelengths include Seaquist \& Bignell (1976), Condon (1983), Klein \& Gräve (1986) (1.2 cm [24.5 GHz] data only), Israel \& de Bruyn (1988), and Lisenfeld et al. (2004). The details of these observations are shown in Table 6; where necessary, we converted sensitivities in brightness temperatures to

sensitivities in $\mathrm{mJy}_{\text {beam }}{ }^{-1}$ using the given frequencies and beam parameters. Our observations reach surface brightnesses two (20 cm observations) to eighty ( $13 \mathrm{~cm}$ observations) times fainter than previous observations. Although our resolution is not as high as some previous observations, we do have better $u$-v plane coverage, making our observations sensitive to both compact sources and extended emission.

We have detected radio continuum emission from NGC 1569 at all four observed wavelengths. In Figure 3, we plot the total flux detected by our measurements along with radio continuum flux measurements collected from the literature by Israel \& de Bruyn (1988) and Lisenfeld et al. (2004). Our measured fluxes at $20 \mathrm{~cm}(1.4 \mathrm{GHz})$ and $13 \mathrm{~cm}(2.7 \mathrm{GHz})$ agree well with the measured fluxes from the literature, justifying our assumption not to correct the WSRT observations at these frequencies for the effects of missing short-spacings. The $6 \mathrm{~cm}(4.86 \mathrm{GHz})$ and $3 \mathrm{~cm}(8.46 \mathrm{GHz})$ fluxes from our combined VLA and Effelsberg images are $-9.5 \%(6 \mathrm{~cm})$ and $-8.8 \%(3 \mathrm{~cm})$ less than the measured Effelsberg fluxes. (Note that the low point from the literature at $3 \mathrm{~cm}$ was an interferometer measurement that did not correct for missing short-spacings.) All our observations are at wavelengths greater than or approximately equal to the break in the radio continuum spectrum at approximately $3.75 \mathrm{~cm}$ (8 GHz) first noted by Israel \& de Bruyn (1988) and modeled by Lisenfeld et al. (2004).

Our total intensity images at all four frequencies are shown in Figures 1 and 2. Note that we show the highest resolution image at each frequency, so the beam size varies between the images. At $20 \mathrm{~cm}$, we detect both the bright central regions and the extended diffuse component first seen by Condon (1983) and first discussed by Israel \& de Bruyn (1988). 
There are two bright peaks of emission in the central regions of the galaxy. The brightest peak is on the northwestern side of the disk and the fainter, slightly elongated peak is on the southeastern side of the disk. In our $6 \mathrm{~cm}$, robust $=0$ image, which has the highest resolution of all our images, we resolve the southeastern peak into two sources. Our observations increase the extent of the diffuse component of the galaxy from $\sim 3^{\prime}$ (Israel \& de Bruyn 1988) to $\sim 4^{\prime}$. This corresponds to a physical extent of approximately $4.4 \mathrm{kpc}$ off the plane of the disk (assuming an inclination of $63^{\circ}$; Stil \& Israel 2002), which is extraordinary considering the disk is only about $3^{\prime}(2.9 \mathrm{kpc})$ long in the radio continuum. We also detect the western arm of the galaxy at all observed frequencies, where previous observations only saw it at $20 \mathrm{~cm} 5^{5}$ As one goes from $20 \mathrm{~cm}$ to $3 \mathrm{~cm}$, the morphology of the diffuse component of the radio continuum emission changes from box-shaped to more oval-shaped. At shorter wavelengths, the western arm becomes increasingly prominent and the extent of the radio continuum halo decreases.

Spectral index maps of the observed total intensity radio continuum emission can provide an important diagnostic of the processes producing the radio continuum emission. We define the spectral index, $\alpha$, as $S \propto \nu^{\alpha}$, where $S$ is the source flux and $\nu$ is the frequency; thermal emission has a spectral index of -0.1 and synchrotron emission has a spectral index of $\sim-0.7$. By looking at the observed spectral index, we can get an idea of whether thermal or synchrotron emission dominates in a particular region.

Spectral index maps, however, are significantly affected by small systematic changes in flux. For example, a $5 \%$ change in the flux level in each total intensity map going into the spectral image can change the spectral index by as much as 0.18 . Because of the artifacts remaining in the $20 \mathrm{~cm}$ and $13 \mathrm{~cm}$ images and the systematic uncertainties in creating spectral index maps from data from two different telescopes (especially ones as different as the VLA and the WSRT), we created a spectral index map for NGC 1569 based only on the $6 \mathrm{~cm}$ and $3 \mathrm{~cm}$ data. To make sure that both images sampled the same spatial scale, we used only the interferometric data and restricted the $u-v$ range of the data imaged to 0.74 to 21 $\mathrm{k} \lambda$; this range is the $u-v$ range common to the observations at $13 \mathrm{~cm}, 6 \mathrm{~cm}$, and $3 \mathrm{~cm}]^{6}$ The beam sizes in each image were slightly different because differences in the sampling of the $u-v$ plane in each image. We smoothed both images to the resolution of the image with the largest beam $\left(9.34^{\prime \prime}\right.$ by $\left.7.54^{\prime \prime}\right)$.

Figure 4 shows the spectral indices between the $6 \mathrm{~cm}$ and $3 \mathrm{~cm}$ data. Only spectral

\footnotetext{
5 Klein \& Gräve (1986) saw a hint of this feature in $24.5 \mathrm{GHz}$ single-dish data.

${ }^{6}$ The range common to the $6 \mathrm{~cm}$ and $3 \mathrm{~cm}$ is 0.74 to $29.3 \mathrm{k} \lambda$, which would increase the resolution slightly.
} 
indices where the formal error on $\alpha$ was less than 0.2 were used. The formal error on $\alpha$ is

$$
\sigma_{\alpha}^{2}=\left[\frac{1}{\log \left(\nu_{1} / \nu_{2}\right)}\right]^{2}\left[\left(\frac{\sigma_{1}}{S_{1}}\right)^{2}+\left(\frac{\sigma_{2}}{S_{2}}\right)^{2}\right]
$$

where $S, \nu$, and $\sigma$ are the flux in a pixel, the frequency, and the noise for a particular image and the subscripts denote the first and second images. The values in our final spectral index map agree with those in a spectral index map created using data that was not selfcalibrated, showing that our self-calibration of the $6 \mathrm{~cm}$ and $3 \mathrm{~cm}$ data has not introduced major uncertainties into our spectral index maps.

The spectral indices in the galactic disk are close to thermal with flattest spectral indices offset by $14^{\prime \prime}$ (a little more than a beam) to the north from the main ridge of $3 \mathrm{~cm}$ continuum emission. Since the southern half of the galaxy is pointed toward the observer and the northern half pointed away (Martin et al. 2002), this spectral index asymmetry about the major axis of the disk can be explained as free-free absorption of synchrotron emission from behind the disk by the disk itself. Free-free absorption flattens spectral indices (Condon 1992). In general, as one moves along the minor axis of the galaxy, away from the disk, the spectral indices become steeper, eventually reaching values of approximately -1 at the southern edge of the galaxy. There are, however, two filamentary regions of flat spectral indices extending to the outer edge of the spectral index map. These flat spectrum filaments are aligned with the outer edges of an $\mathrm{H} \alpha$ bubble (Bubble B in the terminology of Westmoquette et al. 2008, see also Figure 7). The spectral index trends seen here agree with the general trends seen in the higher resolution data of Lisenfeld et al. (2004) supporting their theory that the break in the total non-thermal emission spectrum of NGC 1569 is due to the convective transport of cosmic rays into the halo.

\subsection{Polarized Emission}

The observations presented in Figures 5 and 6 are the first published observations of the polarized emission of NGC 1569 . We detect polarization at $20 \mathrm{~cm}\left(36\right.$ to $\left.80 \mu \mathrm{Jy} \mathrm{beam}^{-1}\right)$, $13 \mathrm{~cm}\left(49 \mu \mathrm{Jy}_{\mathrm{beam}}{ }^{-1}\right.$ to $\left.83 \mu \mathrm{Jy}_{\text {beam }^{-1}}\right), 6 \mathrm{~cm}\left(30\right.$ to $\left.108 \mu \mathrm{Jy}_{\text {beam }}{ }^{-1}\right)$, and $3 \mathrm{~cm}(31$ to $\left.137 \mu \mathrm{Jy} \mathrm{beam}^{-1}\right)$. At $3 \mathrm{~cm}$ and $6 \mathrm{~cm}$, there is polarized emission over a large portion of the galaxy, while at $20 \mathrm{~cm}$ the polarized emission is much spottier and at $13 \mathrm{~cm}$ there are only a few small patchies of polarized emission. Note that the strongest polarization detections at 6 and $3 \mathrm{~cm}$ were with the images with $13^{\prime \prime}$ resolution, i.e., similar resolutions to the $20 \mathrm{~cm}$ data. Figures 5 and 6 show the distribution and direction (E-vectors) of the 
polarized intensity at the observed wavelengths.7 To correct for the bias in the estimated polarized intensity, we use the POLCO task in AIPS with a signal-to-noise cut of three. This signal-to-noise cut ensures that all polarization vectors are significant at the $99 \%$ confidence level (Simmons \& Stewart 1985; Vaillancourt 2006). Decreasing the signal-to-noise cut to two did not add appreciably more vectors. Despite NGC 1569's position near the Galactic plane, $(l, b)=(143.7,11.2)$, there is fortunately very little foreground Faraday rotation along this line of sight. From the Galactic Faraday rotation map of Johnston-Hollitt et al. (2004), we estimate that the foreground Faraday rotation is approximately $+10 \mathrm{rad} \mathrm{m}^{-2}$. Examining the polarized sources in our $20 \mathrm{~cm}$ and $13 \mathrm{~cm}$ data (unfortunately, the only polarized source in our $6 \mathrm{~cm}$ and $3 \mathrm{~cm}$ data is NGC 1569 itself), we find an average rotation measure between the $20 \mathrm{~cm}$ and $13 \mathrm{~cm}$ data of $-8.7 \mathrm{rad} \mathrm{m}^{-2}$ with a standard deviation of $36.7 \mathrm{rad} \mathrm{m}^{-2}$, which agrees with the value from the Johnston-Hollitt et al. (2004) map within the errors.

The polarized emission at $20 \mathrm{~cm}$ is shown in the left hand panel of Figure 5. The percent polarization in this image ranges from $2 \%$ at the center to $70 \%$ at the edges. The central region of NGC 1569 is bright enough to have significant polarized emission resulting from residual instrumental leakages rather than emission intrinsic to the galaxy. Based on measurements of unpolarized sources in the field, we estimate this leakage at approximately $1 \%$. Therefore, we require the polarized emission at $20 \mathrm{~cm}$ to have a fractional polarization of greater than $1 \%$ as well as a signal-to-noise ratio of three to be considered significant. The emission in the final image could be depolarized if the polarization vectors rotate significantly over the observing frequencies that are combined to produce the final image; this effect is known as bandwidth depolarization. To determine if the $20 \mathrm{~cm}$ data is affected, we imaged the first and last intermediate frequencies (IFs) separately (see Table 1 for a summary of our observing setup). These two IFs are each $10 \mathrm{MHz}$ wide and $70 \mathrm{MHz}$ apart. Comparing the polarization vectors for the two IFs, we do not see any rotation of the polarization vectors greater than a few degrees. Therefore, creating the final image by combining the data from all the IFs has not resulted in significant depolarization.

The major polarization features at $20 \mathrm{~cm}$ are associated with $\mathrm{H} \alpha$ features in the galaxy. Figure 7 shows the positions of the various $\mathrm{H} \alpha$ bubbles that have been identified in the literature. The $\mathrm{H} \alpha$ emission is included in both Figure 5 and 7 as a point of comparison between the figures. There are polarization features on the northwest and southwest edges of the disk which correspond to the bases of bubbles B and A. Moving northwest away from the disk there are regions of significant polarized emission that outline the edges of bubble $\mathrm{B}$ as well as some that may be associated with the region of $\mathrm{H} \alpha$ filaments just to the east of

\footnotetext{
${ }^{7}$ We plot E-vectors in Figures 5 and 6 because they are the quantity actually measured by the observations. We rotate the E-vectors by $90^{\circ}$ to estimate the direction of the magnetic field in Figure 10
} 
this feature. We also see significant polarized emission outlining the bubble A.

The $13 \mathrm{~cm}$ emission is shown in the right panel in Figure 5. The polarization ranges from $1.4 \%$ for vectors near the center of the image to $42.1 \%$ for the clump of significant vectors in the northwestern portion of the galaxy. We used one of the calibration sources (3C147) to estimate the residual instrumental leakages at $13 \mathrm{~cm}$ as $0.5 \%$ and eliminated all vectors with smaller percent polarizations. This cut affected only one vector located on the brightest peak of $13 \mathrm{~cm}$ emission. There is very little significant polarization in the $13 \mathrm{~cm}$ data besides the polarization vectors in the northwestern portion of NGC 1569 that correspond to locations of significant polarization vectors at $20 \mathrm{~cm}$. The lack of significant polarized emission is not surprising since the $13 \mathrm{~cm}$ has the highest noise level of all our data. When the $20 \mathrm{~cm}$ polarized intensity is calculated assuming a noise cutoff similar to that of the $13 \mathrm{~cm}$ data we see little polarization, which confirms that the lack of polarization at $13 \mathrm{~cm}$ is most likely the result of the relatively poor sensitivity of this data. To make sure this is not the result of bandwidth depolarization, we have imaged first and last $20 \mathrm{MHz}$ IFs by themselves and compared polarized emission in the two images. Neither one of the single IF images shows any significant polarization, so significant polarization in one IFs is not canceling out significant polarization in another IF.

The most polarized emission is seen in the $6 \mathrm{~cm}$ and $3 \mathrm{~cm}$ data (Figure 6). The percent polarization seen in the $6 \mathrm{~cm}$ and $3 \mathrm{~cm}$ data is well above the estimated leakage of the VLA $(<1 \%$ near the center of the field; Ulvestad \& Taylor 2007). Therefore, the only cut we have applied to this data is to require the signal-to-noise ratio for the polarized emission to be greater than three. At both $6 \mathrm{~cm}$ and $3 \mathrm{~cm}$, there is no visible rotation of the polarization vectors between the two IFs, so bandwidth depolarization does not seem to be an issue. There are some significant polarization vectors outside the $3 \sigma$ contour line for the $3 \mathrm{~cm}$ total intensity. For the $3 \mathrm{~cm}$ data with the $13^{\prime \prime}$ resolution, however, the error in the Stokes I image is larger than estimates of the theoretical noise in Stokes I based on the noise in the Stokes $\mathrm{Q}$ and $\mathrm{U}$ images. Therefore, we are relatively more sensitive to polarized emission than to total intensity emission.

In general, the polarized emission at $3 \mathrm{~cm}$ is strong and present over a large fraction of the galaxy. The polarized emission at this wavelength goes from 1-2\% at the center to up to $40 \%$ and greater on the western edge and $10-30 \%$ in the halo of the galaxy. There is little and/or weak polarized emission coincident with the western $\mathrm{H} \alpha$ arm and another break in the emission at approximately $04^{\mathrm{h}} 30^{\mathrm{m}} 45^{\mathrm{s}}, 64^{\circ} 50^{\prime} 20^{\prime \prime}$. The central regions of the $3 \mathrm{~cm}$ data appear to be largely unpolarized except for some polarized emission associated with the main radio continuum emission peak. This depolarization could be due either to Faraday depolarization or to an inherently turbulent field. We discuss these possibilities further in 
$\S 5$ in the context of NGC 1569's H $\alpha$ kinematics. The majority of the polarized emission is in the southern half of the galaxy, which is unsurprising given that X-ray observations have found that the southern portion of the galaxy is inclined towards us and the northern part is inclined away (see Figure 13 in Martin et al. 2002). The polarized emission from the northern half of the galaxy has a longer path length through ionized gas and thus is subject to more internal depolarization. There is particularly strong polarization on the western edge of the $\mathrm{H} \alpha$ arm. This feature is also seen in higher resolution images not included here. The region on the interior of the $\mathrm{H} \alpha$ arm (Bubble A in Figure 7) is polarized as well as the base of bubble $\mathrm{F}$ to the southeast of the disk. In the northern half of the galaxy, there is polarized emission on the northeastern edge corresponding to Bubble E and to the north of the main radio continuum peak, which corresponds to Bubble B.

The polarized emission at $6 \mathrm{~cm}$ is patchier than at $3 \mathrm{~cm}$ and ranges from $1-2 \%$ in the inner regions of the galaxy to $15-20 \%$ in the outer regions of the galaxy to greater than $40 \%$ in the polarized arm. Interestingly, the E-vectors in common between the $3 \mathrm{~cm}$ and $6 \mathrm{~cm}$ data show little sign of Faraday rotation except for the polarization in the northern half of the galaxy where there is significant rotation (see $\S 4.3$ ). There is significantly more polarized emission north of the plane of the galaxy at $6 \mathrm{~cm}$ than at $3 \mathrm{~cm}$. Since emission at longer wavelengths tends to trace emission at smaller Faraday depths, this emission is probably closer to the observer along the line of sight than the emission at $3 \mathrm{~cm}$. At $6 \mathrm{~cm}$ there, is still polarized emission surrounding the $\mathrm{H} \alpha$ arm as well as polarized emission at the base of Bubble $\mathrm{F}$ in a region of extensive $\mathrm{H} \alpha$ filaments and in the Bubble $\mathrm{E}$ region. These regions of emission, however, are less extended than those seen in the $3 \mathrm{~cm}$ data. The central region of NGC 1569 at $6 \mathrm{~cm}$ has no significant polarization.

In short, our observations reveal that more polarized emission is seen in NGC 1569 at shorter wavelengths than at longer wavelengths and that the polarized emission is associated with $\mathrm{H} \alpha$ bubbles and filaments in the galaxy. The first result is common to radio continuum polarization observations of other galaxies (Beck et al. 1996). Depolarization due to Faraday dispersion and differential Faraday rotation increases with increasing wavelength, so radio continuum polarization observations of galaxies at longer wavelengths show less polarization. We defer discussion of the second result to $\S 4.3$. 


\section{Magnetic Field Structure and Strength}

\subsection{Estimating the Synchrotron Flux}

To determine the magnetic field strength in NGC 1569 and thus assess the relative importance of the field to the ISM, we must estimate the portion of the total radio continuum flux due to synchrotron emission and the portion due to free-free emission. To do this, we estimated the thermal component of the radio continuum emission using the $\mathrm{H} \alpha$ emission from the galaxy. We used a flux calibrated $\mathrm{H} \alpha$ image kindly provided by Deidre Hunter (Hunter \& Elmegreen 2004) to determine the $\mathrm{H} \alpha$ flux in each pixel. We corrected the resulting $\mathrm{H} \alpha$ flux for Galactic extinction using a value of $A_{H \alpha}=1.26$ (Relaño et al. 2006). Since the internal extinction of NGC 1569 is small $\left(A_{H \alpha}<0.6 \mathrm{mag}\right)$ and largely confined to the central regions of the galaxy (Relaño et al. 2006), we do not attempt to correct for internal extinction. Not correcting for internal extinction will cause us to underestimate the $\mathrm{H} \alpha$ flux by, at most, a factor of 1.74 in the regions with the greatest extinction, which are the region surrounding the central peak and the region connecting the two brightest radio continuum peaks (see Figure 9 of Relaño et al. 2006). Note that we do not need to take into account non-photoionization processes since the calculation of the conversion between $\mathrm{H} \alpha$ emission and free-free emission does not depend on the ionization source.

We calculated the expected thermal emission at our observing frequencies from the given $\mathrm{H} \alpha$ flux using the expressions given in Appendix A of Hunt et al. (2004) ${ }^{8}$ which explicitly include the contribution of ionized helium:

$$
\left(\frac{f_{\nu}}{\mathrm{mJy}}\right)=1.16\left(1+\frac{n\left(H e^{+}\right)}{n\left(H^{+}\right)}\right)\left(\frac{T}{10^{4} \mathrm{~K}}\right)^{0.617}\left(\frac{\nu}{\mathrm{GHz}}\right)^{-0.1}\left(\frac{f_{H \alpha}}{10^{-12} \mathrm{erg} \mathrm{cm}^{-2} \mathrm{~s}^{-1}}\right) .
$$

In Equation (2), $f_{\nu}$ is the radio continuum flux, $n\left(H e^{+}\right) / n\left(H^{+}\right)$is the ratio of ionized helium to ionized hydrogen, $T$ is the temperature of the region, $\nu$ is the frequency of the radio continuum observations, and $f_{H \alpha}$ is the Galactic-extinction-corrected $\mathrm{H} \alpha$ flux. We use a $n\left(H e^{+}\right) / n\left(H^{+}\right)$ratio of 0.087 (Martin \& Kennicutt 1997). The expressions from Hunt et al. (2004) are valid for temperatures between $10,000 \mathrm{~K}$ and $20,000 \mathrm{~K}$ and densities between $100 \mathrm{~cm}^{-3}$ and $1000 \mathrm{~cm}^{-3}$. We assume a temperature of $10,000 \mathrm{~K}$, which is typical of HII regions. This temperature is $10 \%$ lower than the lowest temperature that Devost et al. (1997) was able to measure for selected regions of NGC 1569. If we increase the temperature to $11,100 \mathrm{~K}$, this increases the estimated thermal emission by only $7 \%$, since the temperature dependence in this equation goes as $T^{0.617}$. Westmoquette et al. (2008) shows that the densities of ionized gas in NGC 1569 range from $100 \mathrm{~cm}^{-3}$ (the lowest density probed by the

8 See also Caplan \& Deharveng $\sqrt{1986)}$ and Condon $(1992)$. 
their diagnostic) to $1000 \mathrm{~cm}^{-3}$. We smoothed the result of Equation (2) to the resolution of the observations using the AIPS task CONVL. The synchrotron flux is

$$
F_{n t h}=F_{t o t}\left(1-\frac{F_{t h}}{F_{t o t}}\right)
$$

where $F_{n t h}$ is the flux of the synchrotron (non-thermal) emission, $F_{t o t}$ is the total flux observed, and $F_{t h}$ is the thermal flux calculated using Equation (2).

We plot the estimated thermal fraction $\left(F_{t h} / F_{t o t}\right)$ in Figure 8 . Note that we set thermal fractions greater than one to one and thermal fractions less than zero to zero. This does not significantly change the resulting images and only affects regions where the emission is faint, i.e., the edges of the map. As one would expect, the total emission at both $20 \mathrm{~cm}$ and $13 \mathrm{~cm}$ is dominated by synchrotron emission, i.e., has a low thermal fraction, with the maximum thermal fraction $(40 \%)$ southeast of the location of the radio continuum peak. The halo of the galaxy has significantly more synchrotron (less thermal) emission than the disk. As we go to shorter wavelengths, the amount of thermal emission increases to $50 \%$ in the region southeast of the radio continuum peak. Note that the inner portion of the western arm has a significant amount of thermal emission, while the outer portion is largely synchrotron emission. The outline of Bubble A appears very clearly in the $3 \mathrm{~cm}$ map of the thermal emission, which provides additional confirmation that we are able to accurately determine the location of the thermal flux with the procedure described earlier in this section. The entirely thermal regions on the northern and eastern edges of the $3 \mathrm{~cm}$ map are due to the $3 \mathrm{~cm}$ diffuse emission being slightly underestimated there. Although we have been very careful about reconstructing most of the diffuse flux, it is impossible to reconstruct it all, which results in the thermal fraction being overestimated in these regions.

\subsection{Magnetic Field Strength}

The intensity of the non-thermal (synchrotron) emission can be used to estimate the magnetic field strength in a galaxy. The intensity of synchrotron emission at a particular frequency $\left(I_{\nu}\right)$ goes as

$$
I_{\nu} \propto l N_{0} B_{\perp}^{1-\alpha} \nu^{\alpha}
$$

where $l$ is the line of sight through the source, $N_{0}$ is the scaling factor for the electron energy spectrum $\left(N(E) \mathrm{dE}=\mathrm{N}_{0} \mathrm{E}^{-\gamma} \mathrm{dE}, \gamma=1-2 \alpha\right), B_{\perp}$ is the magnetic field strength in the plane of the sky, and $\nu$ is the observing frequency (Ginzburg \& Syrovatskii 1965).9 For a particular value of $I_{\nu}$ only the quantity $N_{0} B_{\perp}^{1-\alpha}$ can be estimated, so a particular synchrotron

\footnotetext{
${ }^{9}$ We use a sign convention for $\alpha$ opposite to the one typically used.
} 
intensity may correspond to a large electron density and a small magnetic field or vice versa. To disentangle the magnetic field strength, one traditionally employs the minimum energy assumption where the total energy (the energy of the magnetic field plus the energy of the cosmic ray electrons plus the energy of the cosmic-ray protons) is minimized. For the typical range of $\gamma$, the minimum energy is similar to the energy derived assuming equipartition between cosmic rays and the magnetic field (Beck \& Krause 2005). The minimum energy/equipartition calculation requires a further assumption that the energy spectrum of the cosmic-ray protons is the energy spectrum of the cosmic-ray electrons scaled by a constant. However, this is not true in the Milky Way, where the electron spectrum is steeper than the proton spectrum (see Figure 1 in Hillas 2006). Beck \& Krause (2005) present a revised equipartition estimate of the magnetic field strength that uses the number density ratio of electrons and protons rather than the more typical energy density ratio to estimate the field strength. They use the equation below (Equation 3 in Beck \& Krause 2005) to estimate the total equipartition magnetic field strength:

$$
B_{t}=\left[\frac{4 \pi(1-2 \alpha)\left(K_{0}+1\right) I_{\nu} E_{p}^{1+2 \alpha}\left(\nu / 2 c_{1}\right)^{-\alpha}}{(-2 \alpha-1) c_{2} l c_{4}(i)}\right]^{1 /(3-\alpha)}
$$

where $B_{t}$ is the total equipartition magnetic field in $\mathrm{G}, K_{0}$ is the number density ratio of protons to electrons, $E_{p}$ is the rest energy of the proton, $c_{1}$ is a constant equal to $6.26428 \times$ $10^{18} \mathrm{erg}^{-2} \mathrm{~s}^{-1} \mathrm{G}^{-1}, c_{2}$ is a constant tabulated on page 232 of Pacholczyk (1970) as $c_{5}, l$ is the path length through the galaxy, and $c_{4}(i)$ is a function correcting for the inclination of the region with respect to the sky 10 Beck \& Krause (2005) caution that this formula may not be strictly applicable to starburst galaxies, since synchrotron losses are important in high-density regions, and that detailed modeling is needed to determine the true magnetic field of a starburst. However, based on the work of Thompson et al. (2006), we believe that our magnetic field strength estimates for NGC 1569 will not be significantly underestimated. Thompson et al. (2006) estimate, for a large number of galaxies from normal spirals to ULIRGs, what they call an "equipartition" magnetic field using the assumption that the magnetic energy density is comparable to the gravitational pressure of the ISM and compare it to a minimum energy magnetic field estimated using a method similar to Equation (5). The Thompson et al. "equipartition" field is the largest magnetic field a galaxy can have and still be gravitationally stable object. In Thompson et al. Figure 1, NGC 1569 lies on the locus where both the "equipartition" magnetic field and the minimum energy magnetic field are similar, far from the position of galaxies like NGC 253, M82, and Arp 220, whose minimum energy magnetic field estimates are well below the Thompson et al. "equipartition" field.

10 We use the opposite sign convention for $\alpha$ as Beck \& Krause (2005). 
We use the method of "magnetic maps" described in Chyży (2008) to make a map of the total $\left(B_{t}\right)$, uniform $\left(B_{u}\right)$, and random $\left(B_{r}\right)$ magnetic field strengths as functions of position in the galaxy along with the revised equipartition equations given in Beck \& Krause (2005) to calculate the magnetic field strength. Equation (5) assumes that the magnetic field is either all uniform or all random. Since the magnetic field of NGC 1569 is clearly a mixture of the two in many regions, we would like to derive a version of Equation (5) that accounts for both the uniform and random field components. Equation (5) is derived from the more general Equation A.16 in Beck \& Krause (2005), which gives the cosmic ray energy density. By equating the magnetic field energy density $\left(\epsilon_{B}=B_{t}^{2} / 8 \pi\right)$ to the cosmic ray energy density $\left(\epsilon_{C R}\right)$, one obtains

$$
\begin{aligned}
\epsilon_{B} & =\epsilon_{C R} \\
\frac{B_{t}^{2}}{8 \pi} & =\frac{(1-2 \alpha)\left(K_{0}+1\right) I_{\nu} E_{p}^{1+2 \alpha}\left(\nu /\left(2 c_{1}\right)\right)^{-\alpha}}{2(-1-2 \alpha) c_{2} l B_{\perp}^{(1-\alpha)}} \\
B_{t}^{2} & =\frac{4 \pi(1-2 \alpha)\left(K_{0}+1\right) I_{\nu} E_{p}^{1+2 \alpha}\left(\nu /\left(2 c_{1}\right)\right)^{-\alpha}}{(-1-2 \alpha) c_{2} l B_{\perp}^{(1-\alpha)}}
\end{aligned}
$$

Note that $\alpha$ here equals $\gamma=1-2 \alpha$ in Beck \& Krause (2005) and that $c_{2}$ is a function of $\alpha$. Since Equation 8 includes $B_{t}, B_{\perp}$, and $l$, it not only needs to take into account the geometry of NGC 1569 and its associated magnetic field, but also the contribution to the total field from both the uniform and random components. We model the geometry of NGC 1569 as an infinite cylinder rotated on the plane of the sky and inclined with respect to the line of sight. Appendix A.1 gives an equation for the line of sight distance through the galaxy. The geometrical correction to obtain the total field from the observed field is more complex. For an isotropic random field, the correction factor $\left(c_{4}\right)$ is

$$
c_{4}=\left(\frac{B_{r, \perp}}{B_{r}}\right)^{(1-\alpha)}=\left(\frac{2}{3}\right)^{(1-\alpha) / 2}
$$

For the regions of NGC 1569 where we do not see any polarization, we assume that the field is completely random. In this case, the field strength is given by Equation (5) with the geometrical correction factor given by Equation (9). For a completely uniform field,

$$
c_{4}=\left(\frac{B_{u, \perp}}{B_{u}}\right)^{(1-\alpha)}=\left[\left(\sin \left(\mathrm{PA}_{\mathrm{B}}\right)\right)^{2}+\left(\frac{\cos \left(\mathrm{PA}_{\mathrm{B}}\right)}{\sin i}\right)^{2}\right]^{-(1-\alpha) / 2}
$$

where $\mathrm{PA}_{\mathrm{B}}$ is the position angle of the magnetic field on the sky. For the rest of this paper, $c_{4}$ will refer to the version for a completely uniform field. For a derivation of this quantity, see Appendix A.2. 
We cannot assume that regions of polarized emission are completely uniform; there will still be a contribution of the random component in these regions. To deal with this, we first need to estimate the ratio, $q$, of the random magnetic field $\left(B_{r}\right)$ to the uniform magnetic field in the plane of the sky $\left(B_{u, \perp}\right)$. To estimate the ratio $q$, we use the observed percent polarization $(p)$ and the relation from Sokoloff et al. (1998)

$$
p=p_{0} \frac{1+(7 / 3) q^{2}}{1+3 q^{2}+(10 / 9) q^{4}}
$$

where $p_{0}$ is the intrinsic polarization of synchrotron emission $\left(p_{0}=(3-3 \alpha) /(5-3 \alpha)\right) .^{11}$ Equation (11) is derived assuming that the cosmic rays are coupled to the magnetic field (Sokoloff et al. 1998; Beck et al. 2003). The more commonly used version of this formula (Burn 1966)

$$
p=p_{0} \frac{1}{1+\frac{2}{3} q^{2}}
$$

does not include this assumption. Both the Equations (11) and (12) assume isotropic fluctuations. Using $q$, one can now recast the total magnetic field $B_{t}$ in terms of the $B_{r}$ and $B_{u}$. Using the relations $B_{t}^{2}=B_{r}^{2}+B_{u}^{2}, B_{\perp}^{2}=B_{r, \perp}^{2}+B_{u, \perp}^{2}$ and the geometrical parameters given in Equations 9 and 10 ,

$$
\begin{aligned}
B_{r, \perp}^{2} & =\frac{2}{3} B_{r}^{2} \\
B_{u, \perp} & =B_{u} c_{4}^{1 /(1-\alpha)} \\
B_{r} & =q B_{u, \perp}
\end{aligned}
$$

so

$$
\begin{aligned}
B_{t}^{2} & =\left(q^{2} c_{4}^{2 /(1-\alpha)}+1\right) B_{u}^{2} \\
B_{\perp}^{2} & =\left(\frac{2}{3} q^{2}+1\right) c_{4}^{2 /(1-\alpha)} B_{u}^{2} \\
B_{\perp}^{(1-\alpha)} & =\left(\frac{2}{3} q^{2}+1\right)^{(1-\alpha) / 2} c_{4} B_{u}^{1-\alpha}
\end{aligned}
$$

Substituting the above expressions for $B_{t}^{2}$ and $B_{\perp}^{(1-\alpha)}$ into Equation (8), we obtain an expression giving uniform field strength in a region where we have both a uniform and random field

$$
B_{u}=\left[\frac{4 \pi(1-2 \alpha)\left(K_{0}+1\right) I_{\nu} E_{P}^{1+2 \alpha}\left(\nu /\left(2 c_{1}\right)\right)^{-\alpha}}{(-1-2 \alpha) c_{2}(\alpha) l c_{4}\left((2 / 3) q^{2}+1\right)^{(1-\alpha) / 2}\left(q^{2} c_{4}^{2 /(1-\alpha)}+1\right)}\right]^{1 /(3-\alpha)}
$$

11 Beck \& Krause (2005)'s version of this equation has a sign error, cf. Ginzburg \& Syrovatskii (1965). 
In the regions where we have both a random and uniform component to the field, we calculate $q$ using Equation (11) and then calculate $B_{u}$ from Equation (19) using the total synchrotron emission and the value of $c_{4}$ determined from the polarization angle of the emission using Equation 10. The total and random fields can then be derived from the values of $B_{u}, q$, $c_{4}$, and $\alpha$.

$$
\begin{aligned}
& B_{r}=q B_{u} c_{4}^{1 /(1-\alpha)} \\
& B_{t}=\left(B_{r}^{2}+B_{u}^{2}\right)^{1 / 2}
\end{aligned}
$$

For the other parameters in Equations 19, 20, and 21, we determined the position angle and center of the disk by fitting ellipses to the total intensity radio continuum image at $3 \mathrm{~cm}$ using the IRAF (Tody 1986, 1993) package ellipse. The position angle of the disk is $-62.1^{\circ}$, which corresponds to an angle $(\beta)$ between the plane of the disk and the RA axis of $27.9^{\circ}$, and the center of the galaxy is $\left(4^{\mathrm{h}} 30^{\mathrm{m}} 49^{\mathrm{s}} 25,64^{\circ} 50^{\prime} 53^{\prime \prime} \cdot 0\right)$. The diameter of the disk of NGC 1569 was estimated from the $3 \mathrm{~cm}$ image to be $2.1 \mathrm{kpc}$. We assume that the distance and inclination of the galaxy are 3.36 Mpc (Grocholski et al. 2008) and $63^{\circ}$ (Stil \& Israel 2002), respectively. Since even small errors in the determination of the non-thermal fluxes of individual pixels will correspond to large errors in $\alpha .2^{12}$ we estimate a value for $\alpha$ by calculating $\alpha$ for the total non-thermal flux at $6 \mathrm{~cm}$ and $3 \mathrm{~cm}$. This method gives a value of $\alpha=-0.97$. We take $K_{0}$ to be 100 , which is a typical value in the solar neighborhood, but which may be as low as 40 in regions of strong shocks (Beck \& Krause 2005). Combining all these values, we finally determine $B_{u}, B_{r}$, and $B_{t}$ for each pixel in the $3 \mathrm{~cm}$ maps using Equations 19, 20, and 21.

Our magnetic maps based on the $3 \mathrm{~cm}$ images with $13^{\prime \prime}$ resolution are shown in Figure 9 . The maximum total magnetic field strength corresponds to the peak in radio continuum emission at $3 \mathrm{~cm}$ and reaches a value of $38 \mu \mathrm{G}$. In the extended halo of the galaxy, the total magnetic field has a value of $10-15 \mu \mathrm{G}$. Examining the plot of $q$ values, i.e., the ratio of the random field to the uniform field in the plane of the sky, we see that $q$ is high $(\sim 20)$ in the central regions of the galaxy, suggesting that the random field dominates in these regions, although there could be significant Faraday depolarization in this region due to the high electron density. In the outer edges, however, $q$ is only $1-2$, so the regular field is quite strong in these regions. This effect is also seen in the magnetic fields of spiral galaxies (Heiles 1996). The regular field in the synchrotron arm ranges between $9 \mu \mathrm{G}$ at the southern tip to about $3 \mu \mathrm{G}$ where it connects up with the disk of the galaxy.

To get an idea of how the uncertainties in our parameter estimates affect our estimated

\footnotetext{
${ }^{12}$ Here $\alpha$ represents the spectral index of the non-thermal emission.
} 
magnetic field strength, we varied each parameter individually by the estimated error in each parameter, calculated the difference between the new magnetic field estimate and the nominal estimate shown in Figure 9, and finally added the differences from each parameter variation in quadrature. We varied $\alpha$ by $-0.3, K_{0}$ to 40 (a value typical for strong shocks), the distance by $10 \%$, the diameter by $20 \%$, the position angle by $3^{\circ}$, the center by $6^{\prime \prime}$ in each direction, and the inclination by $3^{\circ}$. The resulting calculation showed that the uncertainties on the total magnetic field strength in the central region and the outer region of the galaxy were $10 \mu \mathrm{G}$ and $3-4 \mu \mathrm{G}$, respectively. This error is almost entirely due to the error in the random field. The error in the determination of the uniform field is only $0.5-2 \mu \mathrm{G}$. If the Burn (1966) formula for $q$ is used instead of the Sokoloff et al. (1998) formula for $q$, the uniform magnetic field is larger by a factor of 1.2 in the center of the galaxy and remains roughly the same at the edge and the random field remains the same everywhere.

We also investigated how systematic uncertainties in the estimation of the synchrotron flux due to internal extinction in the central region of the galaxy will affect the estimated magnetic field. Assuming that the maximum internal extinction of 1.74 holds over the entire galaxy, we get a synchrotron flux down by a factor 0.6 near the main radio continuum peak, 0.5 near the secondary radio continuum peak, and 0.2 in the region between the two peaks. Since the radio continuum emission is entirely random in the central region,

$$
B_{t} \propto I_{\nu}^{1 /(3-\alpha)}
$$

and using the value of $\alpha$ determined above (-0.97), the maximum factor by which the magnetic field strength will be decreased is 0.88 for the main radio continuum peak, 0.84 for the secondary radio continuum beam, and 0.67 for the region between the two peaks.

\subsection{Magnetic Field Structure}

The structure of NGC 1569's magnetic field reveals important clues about the interaction of the magnetic field with the other components of the interstellar medium and about the mechanisms at work in the galaxy that may have amplified the field. From our data set,

we have two complementary measures of the structure of the magnetic field. In Figure 10 , the $3 \mathrm{~cm}$ polarization vectors rotated by $90^{\circ}$ give an estimate of the orientation, but not direction, of the magnetic field in the plane of the sky. There is very little Faraday rotation at $3 \mathrm{~cm}$, so the estimate of the magnetic field orientation at $3 \mathrm{~cm}$ should be accurate. The difference in the position angle of the polarized emission at different wavelengths gives the rotation measure

$$
\Delta \phi=\mathrm{RM} \lambda^{2}
$$


which depends on the thermal electron density and the magnetic field along the line of sight

$$
\mathrm{RM}=0.812 \int B_{\|} n_{e} \mathrm{~d} s,
$$

where RM is in $\mathrm{rad} \mathrm{m}{ }^{-2}, n_{e}$ is in $\mathrm{cm}^{-3}, B_{\|}$is in $\mu \mathrm{G}$, and $\mathrm{d} s$ is in pc Gardner \& Whiteoak 1966). The sign of the rotation measure gives the direction, not just the orientation of $B_{\|}$. Positive rotation measures point towards the observer and negative away. In Figure 10, we plot the rotation measures between the $6 \mathrm{~cm}$ and $3 \mathrm{~cm}$ data with the estimated magnetic field vectors from the $3 \mathrm{~cm}$ data overlaid. We do not show the rotation measures between between the $20 \mathrm{~cm}$ and $13 \mathrm{~cm}$ data because there are only two patches of significant rotation measures. These patches are the size of the beam and are located at $4^{\mathrm{h}} 30^{\mathrm{m}} 41^{\mathrm{s}} \cdot 41,64^{\circ} 52^{\prime} 12^{\prime \prime} \cdot 98$ (-36 $\left.\mathrm{rad} \mathrm{m}^{-2}\right)$ and $4^{\mathrm{h}} 30^{\mathrm{m}} 45.17,65^{\circ} 51^{\prime} 52^{\prime \prime} \cdot 0\left(42 \mathrm{rad} \mathrm{m}^{-2}\right)$. The rotation measures were determined using the AIPS task COMB.

Generally, polarization observations at two frequencies do not have enough information to accurately determine rotation measures (Reuter \& Klein 1996). However, examining Figures 5 and 6, we find that the changes in angle within each pair of frequencies are small enough that we are able to determine the rotation measure between the frequencies in each pair with reasonable accuracy. For the polarization vectors to have rotated at least $180^{\circ}$ between $6 \mathrm{~cm}$ and $3 \mathrm{~cm}$, the rotation measure would have to be greater than $1230 \mathrm{rad} \mathrm{m}^{-2}$, which is much larger than the rotation measures seen in other starbursting galaxies (see 4.4.3). Large changes in the distribution of the polarized emission between $3 \mathrm{~cm}$ and $20 \mathrm{~cm}$ that prevent the determination of rotation measures using at least three frequencies are a common feature of magnetic field observations of edge-on starbursting galaxies (Reuter et al. 1994; Beck et al. 1994; Dahlem et al. 1997; Golla \& Hummel 1994).

From Figure 10, one can see from the magnetic field vectors that, in general, the magnetic field in the plane of the sky is roughly perpendicular to the disk of the galaxy. The magnitude of the rotation measures in the northeastern half of the galaxy are larger than those in the southwestern half of the galaxy. The relative magnitude of the rotation measures between these two regions supports the geometry derived from X-ray measurements: the southern half of NGC 1569 is inclined towards us, while the northern half is inclined away (Martin et al. 2002). Therefore, the emission from the region north of the disk passes through a larger column of ionized gas and thus has a correspondingly larger rotation measure. In the southeastern portion of the galaxy, there are more rotation measures close to zero which means that, in general, the field we are seeing is mostly in the the plane of the sky and that the field along the line of sight is small. This overall magnetic field configuration means that the field can help transfer energy and momentum from cosmic rays to the thermal plasma

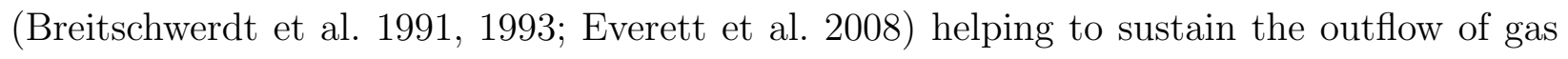
from NGC 1569. 
The magnetic field structure of NGC 1569 is closely tied to the bubbles of ionized gas seen in $\mathrm{H} \alpha$ (see Figure 7 for a finding chart of the $\mathrm{H} \alpha$ bubbles identified in NGC 1569). In Figure 10 in the northeastern portion of the galaxy, we see a pair of oppositely directed rotation measures. The field along the line of sight in the easternmost region is directed toward the observer (yellow/green region in the northeastern part), while the field to the northwest of it is directed away from the observer (the blue region in the northeastern part). These rotation measures coincide with $\mathrm{H} \alpha$ bubble E. The combination of the orientation of the magnetic field vectors in the plane of the sky with the observed rotation measures suggests a magnetic loop being drawn out of the disk. The magnetic field vectors at $20 \mathrm{~cm}$ (see Figure 5 for the polarization vectors, which can be rotated by $90^{\circ}$ to get an estimate of the magnetic field vectors) and the two small regions of significant rotation measures between the $20 \mathrm{~cm}$ data and the $13 \mathrm{~cm}$ mentioned earlier are associated with $\mathrm{H} \alpha$ Bubble B. The sign of the rotation measures between the $20 \mathrm{~cm}$ and $13 \mathrm{~cm}$ data is oppositely directed in the two regions, suggesting another field loop especially given the estimated orientation of the magnetic field in the plane of the sky.

Looking closely at the rotation measures between $6 \mathrm{~cm}$ and $3 \mathrm{~cm}$ in the southern portion of the galaxy, one can see indications of other possible magnetic field loops associated with $\mathrm{H} \alpha$ bubbles $\mathrm{A}$ and $\mathrm{F}$. The southern patch of rotation measures at $4^{\mathrm{h}} 30^{\mathrm{m}} 50^{\mathrm{s}}$ shades from blue to green, which means that the field along the line of sight is going from being pointed away from the observer to pointed toward the observer. This region is associated with Bubble F. The patch of rotation measures at $4^{\mathrm{h}} 30^{\mathrm{m}} 42^{\mathrm{s}}$ shows the opposite trend. The field goes from being almost completely in the plane of the sky (green) to pointed away from the observer (blue). The western arm goes from yellow where it connects to the disk of the galaxy (field pointed towards observer) to green (field primarily in the plane of the sky) to blue-green (field pointed away from observer). These regions are both associated with Bubble A.

The bases of all the bubbles are around 30" which at the distance of NGC 1569 is a physical size of about 500 pc. This size is roughly double the size of the based of an average superbubble in the Milky Way (200 pc; Ferrière 2001).

\subsection{Implications of Derived Magnetic Field Strength and Structure}

\subsubsection{Role of the Field in NGC 1569}

From our magnetic field estimates, we can calculate a typical pressure for the central region and halo of NGC 1569. Adopting $38 \mu \mathrm{G}$ for the central field and $12 \mu \mathrm{G}$ for the halo field (see Figure 9), we get pressures $(P / k)$ of $4.2 \times 10^{5} \mathrm{~K} \mathrm{~cm}^{-3}$ and $0.41 \times 10^{5} \mathrm{~K} \mathrm{~cm}^{-3}$ 
for the central magnetic pressure and the halo magnetic pressure. In Table 7, we compare these values with the pressures determined for other components of NGC 1569's ISM and the overall gravitational pressure. Where necessary, we have corrected the input values for the updated distance to NGC 1569. Except for the pressure of the diffuse ionized medium (DIG), we have not included any corrections for the highly uncertain filling factors of various components 13 Note that we associate the broad component of the turbulent pressure with the DIG and the narrow component with the HII regions. Table 7 shows that the magnetic pressure in NGC 1569 is generally the same order of magnitude as most of the other sources of pressure in its ISM, but is also generally less than the other pressures in similar regions. The dominant pressure source in NGC 1569 is actually the turbulent pressure of the HII shells, which is not surprising given the level of disruption in this galaxy. The central x-ray pressure is almost as large as the gravitational pressure which explains the extended halo of hot gas around NGC 1569. The pressure balance in NGC 1569 is very different from the pressure balances in NGC 6946 (Beck 2007) and M33 (Tabatabaei et al. 2008). For these galaxies, the energy densities (i.e. pressures) of the magnetic field and the turbulent component are roughly comparable, while the pressure for the thermal gas is down by a factor of approximately 5. NGC 6946 and M33, however, are very different galaxies from NGC 1569. They are relatively quiescent, star-forming galaxies, unlike NGC 1569, which is in final throes of a starburst that has significantly affected its ISM.

\subsubsection{Generating the Field}

Ferrière \& Schmitt (2000, and references therein) and Gressel et al. (2008) have developed models in which galactic rotation provides the shear and supernova explosions drive the turbulence needed to generate a large scale magnetic field. In these models, one would expect the magnetic field to be correlated with the supernovae driven bubbles as seen in NGC 1569 (see $\S 4.2$. Note that this is a necessary, but not sufficient condition for a supernova driven dynamo to work. The calculations of Ferrière et al. and Gressel et al. are based on mean field $\alpha-\omega$ dynamo models, which have been criticized on theoretical grounds (Kulsrud \& Zweibel 2008; Cattaneo \& Hughes 2009). Nevertheless, whether or not mean field theory is valid, it seems likely that shear and turbulence are key ingredients in a galactic dynamo. Therefore, we estimate the strength of these two components (turbulence via supernova explosions and shear via differential rotation) for NGC 1569.

\footnotetext{
${ }^{13}$ Decreasing the filling factors from one, which is what we assume here, will increase the pressure by a factor of $1 / \sqrt{f}$.
} 
First, we compare the supernova rate in the Milky Way to that of NGC 1569. The supernova rate from type II supernovae in the Milky Way is $27 \mathrm{Myr}^{-1} \mathrm{kpc}^{-2}$ Ferrière 2001).14 Angeretti et al. (2005) find that the recent star formation rate for NGC 1569 is $6.4 \mathrm{M}_{\odot} \mathrm{yr}^{-1} \mathrm{kpc}^{-2}$. This star formation rate was determined using a distance estimate of $2.9 \mathrm{Mpc}$. Increasing the distance used in the star formation rate estimate to the best current estimate $(3.36 \mathrm{Mpc})$ will increase the rate by a factor of two to three and cause the latest epoch of star formation to end recently (Grocholski et al. 2008). Assuming a Salpeter IMF with 0.8 and $150 \mathrm{M}_{\odot}$ as the minimum and maximum stellar masses and normalizing it to the total number of stars formed per year and per $\mathrm{kpc}^{2}$, we find that that the number of stars formed with masses greater than $8 \mathrm{M}_{\odot}$ for NGC 1569 is $1.1 \times 10^{5} \mathrm{Myr}^{-1} \mathrm{kpc}^{-2}$. With a duration of the most recent burst of star formation of 19 Myr (Angeretti et al. 2005) and a typical lifetime for an $8 \mathrm{M}_{\odot}$ star of $30 \mathrm{Myr}$, we get a supernova rate of $7 \times 10^{4} \mathrm{Myr}^{-1} \mathrm{kpc}^{-2}$. This rate is a factor of 2500 higher than the supernova rate in the Milky Way.

The shear is defined as

$$
\frac{\mathrm{d} \Omega}{\mathrm{dR}}
$$

where $\Omega$ is the angular speed $(v / R)$ and $R$ is the radius. Given that the rotation velocity in the outer regions of galaxy disks is roughly constant, the shear can be estimated by

$$
\left|\frac{\mathrm{d}(v / R)}{\mathrm{d} \mathrm{R}}\right|=\frac{v}{R^{2}} .
$$

In the Milky Way, the angular speed is approximately $220 \mathrm{~km} \mathrm{~s}^{-1}$ at a radius of $8.0 \mathrm{kpc}$, so the shear is $3.4 \mathrm{~km} \mathrm{~s}^{-1} \mathrm{kpc}^{-2}$. In NGC 1569, the galaxy has a rotation speed (corrected for an inclination of $60^{\circ}$ ) of $46 \mathrm{~km} \mathrm{~s}^{-1}$ at a radius of $1.4 \mathrm{kpc}$ (Mühle et al. 2005), which gives a shear of $33 \mathrm{~km} \mathrm{~s}^{-1} \mathrm{kpc}^{-2}$, which is a factor of 10 larger than the shear in the Milky Way.

Since both the shear and the supernovae rate are much greater than in the Milky Way, the fidicual model for this type of dynamo, it is quite possible for a supernova-driven dynamo like those described in Ferrière \& Schmitt (2000) and Gressel et al. (2008) to be at work in NGC 1569. Detailed modeling, however, is needed to determine whether or not this type of dynamo is at work in NGC 1569. Note that the supernova-driven dynamo described above is different that the related mechanism modeled described by Parker (1992) and modeled by Otmianowska-Mazur et al. (2009). In that mechanism, the magnetic buoyancy driven by cosmic rays lofts the field loops into the halo of the galaxy.

\footnotetext{
${ }^{14}$ Including type I supernovae increases the total rate by $15-20 \%$.
} 


\subsubsection{Comparison to Magnetic Fields of Other Starbursts}

Table 8 summarizes the estimated magnetic field strengths in other starburst galaxies. Overall, we find that our estimate for the magnetic field strength of NGC 1569 is in the middle of the distribution of magnetic field strengths for starbursting galaxies with NGC 1569's field being greater than that of NGC 253 and NGC 4666, but less than that of M82 and NGC 1808. It is important to note, however, that these galaxies are all much larger than NGC 1569, so for the field in NGC 1569 to be even comparable to that in larger galaxies has interesting implications for dynamo theory. Thompson et al. (2006) argue that the magnetic field strengths of starbursts like NGC 253, M82, and NGC 1808 are underestimated by the minimum energy magnetic field estimates based on synchrotron emission and argue for much greater fields strengths in starbursting galaxies based on equipartition of the gravitational and magnetic pressures. However, observations of Zeeman splitting in (U)LIRGS by Robishaw et al. (2008), which yield direct measurements of the magnetic field strength, agree roughly with the values derived from synchrotron emission.

Comparing the magnetic field structure we derive for NGC 1569 (see Figure 9) with the magnetic fields structures derived for other starburst galaxies (NGC 253, M82, NGC 1808, NGC 4569, and NGC 4446), we can see several important differences. First, the polarization features in NGC 1569 are patchier than in any of the other galaxies. There are two probable causes for this patchiness: 1) the magnetic field of NGC 1569 is much more turbulent and 2) the depolarization effects in NGC 1569 are much more severe. Given the turbulent nature of the $\mathrm{H} \alpha$ in the central regions of NGC 1569 (Westmoquette et al. 2008), it is likely that the field is very turbulent in this region as well, although the large electron density here makes it possible for Faraday depolarization to mask any uniform field component. However, in the outer regions of this galaxy depolarization effects due to the $\mathrm{H} \alpha$ filaments are likely to dominate over turbulence given the organization imposed on the ISM by the gas outflow. In addition, if the depolarization in the outer regions was due to turbulence it would mean that the turbulence would have to vary in the halo of the galaxy, which is at odds with the result of Westmoquette et al. (2008), who found that the warm ionized gas in the outer regions has more or less constant velocity width between 20 to $70 \mathrm{~km} \mathrm{~s}^{-1}$. The second difference between the previous observations and our observations of NGC 1569 is that the ratio of the length of the polarized arm to the major axis of the radio continuum emission associated with the galactic disk is very high $(\sim 0.5)$. Of the previous observations of starbursting galaxies, only NGC 4569 (Chyży et al. 2006), whose interstellar medium has been shaped by its location in the Virgo cluster, has such a large ratio of radio continuum feature length to length of the major axis of the radio continuum emission associated with the disk.

Rotation measures have been determined for M82 (Reuter et al. 1994), NGC 253 (Beck 
et al. 1994), and NGC 4446 (Dahlem et al. 1997). Comparing our derived rotation measures in Figure 10 to those derived between $6 \mathrm{~cm}$ and $3 \mathrm{~cm}$ for M82 and NGC 253, we see that the average rotation measure between these two frequencies is more or less the same for all three galaxies $\left(\sim \pm 200 \mathrm{rad} \mathrm{m}^{-2}\right)$. The rotation measures for NGC 4666 were determined between $20 \mathrm{~cm}$ and $6 \mathrm{~cm}$ and their magnitudes correspond well to the rotation measures determined here between $20 \mathrm{~cm}$ and $13 \mathrm{~cm}\left(\sim \pm 40 \mathrm{rad} \mathrm{m}^{-2}\right)$.

\section{A Multiwavelength Picture of the Interstellar Medium of NGC 1569}

NGC 1569 is a well-studied galaxy. In this section, we compare our observations with those at other wavelengths to examine the larger picture of the complex interstellar medium of NGC 1569. We start with the $\mathrm{H} \alpha$ emission from NGC 1569, which is characterized by long $\mathrm{H} \alpha$ filaments extending along the minor axis of the galaxy. The filaments were first discussed extensively by Hodge (1974) and were the subject of much subsequent work (e.g. de Vaucouleurs et al. 1974; Waller 1991; Hunter et al. 1993; Heckman et al. 1995; Hunter \& Gallagher 1997). Westmoquette et al. (2007a b) show that in the central region the emission lines have two components: a narrow-line component (FWHM $\leq 70 \mathrm{~km} \mathrm{~s}^{-1}$ ) that they associate with the turbulent ISM and a broad-line component (FWHM $\sim 150 \mathrm{~km} \mathrm{~s}^{-1}$ ) that they associate with emission from a turbulent mixing layer between cool gas clumps and the hot gas from surrounding star clusters. The broad-line component of the emission is confined to the central region of the galaxy (Westmoquette et al. 2008). The outer regions of NGC 1569 show clear signs of an outflow of gas from the center (Westmoquette et al. 2008). This result agrees with previous results from long-slit spectra (Tomita et al. 1994; Heckman et al. 1995; Martin 1998).

Figures 5 and 6 compare the distribution of the $\mathrm{H} \alpha$ emission to the radio continuum emission and were partially discussed in $\S 3.2$ and 4.3 . Here we discuss three additional points. First, the radio continuum arm becomes increasingly aligned with the $\mathrm{H} \alpha$ arm as the wavelength decreases. This fits well with the region on the outside of the $\mathrm{H} \alpha$ arm being synchrotron dominated, which is what one would expect from our thermal fraction estimates in $\S 4.1$. Second, the uniform component of the magnetic field in this region may be at least partially due to compression by the expanding $\mathrm{H} \alpha$ bubble in that region. In Figure 9, the region of uniform magnetic field lies outside of (to the west) of the $\mathrm{H} \alpha$ arm. Finally, the presence of a magnetic field in the $\mathrm{H} \alpha$ arm region may explain why the western $\mathrm{H} \alpha$ arm is thicker than the other $\mathrm{H} \alpha$ features in this galaxy (see Figures 5 and 6). The field here could provide an additional source of pressure, working against any compression forces, thereby increasing the width of the filament compared to the filaments elsewhere in 
the galaxy. However, the effect of the magnetic field on the arm is depends on a variety of factors including the magnetic field strength in the arm, the variation of thermal pressure with height above the galaxy disk, and the effect of the starburst on the distribution of ISM of NGC 1569. In addition, our data only gives the integrated magnetic field - we cannot determine what the field is in the $\mathrm{H} \alpha$ arm, only the integrated field in that region, which is dominated by depolarized effects. Detailed modeling is the only way to disentangle these effects.

X-ray observations of NGC 1569 reveal that the galaxy is surrounded by a halo of hot gas bounded by $\mathrm{H} \alpha$ filaments (Heckman et al. 1995, della Ceca et al. 1996). High spatial resolution observations by Martin et al. (2002) have shown that the bright X-ray emission near the filaments is most likely due to shocks generated by the outflow of gas from NGC 1569. The measured ratio of $\alpha$ elements to iron is between 2.5 and 4.8 times the solar ratio, indicating that the wind is laden with the products of type II supernovae (Martin et al. 2002). We compare our radio continuum observations with the adaptively smoothed X-ray map of Ott et al. (2005a) in Figure 11. To compare the extents of the X-ray and radio continuum maps, we have derived scale lengths for the radio continuum emission by azimuthally averaging the emission as a function of radius from the center of the emission using IRING in AIPS. We find that the scale lengths are $376.1 \mathrm{pc}(20 \mathrm{~cm}), 310.9 \mathrm{pc}(13 \mathrm{~cm})$, $236.5 \mathrm{pc}(6 \mathrm{~cm})$, and $258.7 \mathrm{pc}(3 \mathrm{~cm})$. Ott et al. (2005b) found that the scale length for the $\mathrm{X}$-ray emission is $208 \mathrm{pc}$, so the radio continuum emission extends further than the X-ray emission at all wavelengths. We suggest that the magnetic field in the radio continuum arm may confine the hot gas on the western edge of the galaxy and funnel the gas toward the halo of the galaxy. Similar effects are seen in magnetohydrodynamic simulations of the ISM by de Avillez \& Breitschwerdt (2005).

The neutral hydrogen distribution of NGC 1569 is quite complex (cf. Stil \& Israel 2002; Mühle et al. 2005 and Mühle et al. 2006). The HI distribution has an arm to the west of the galaxy, but this feature is distinct from the arm seen in $\mathrm{X}$-ray, $\mathrm{H} \alpha$, and radio continuum. Mühle et al. (2005) suggest that the HI arm may be the remnant of a primordial gas cloud that interacted with NGC 1569 and started the most recent burst of star formation in this galaxy; the HI arm is likely still in the process of accreting onto the disk of the galaxy. In Figure 12, we compare the neutral hydrogen distribution to our radio continuum observations. The location of the polarized western arm south of the HI arm also suggests an interaction between the infalling HI arm and the galaxy itself. We speculate that the strong magnetic field associated with the $\mathrm{H} \alpha$ arm is the result of the collision of the $\mathrm{HI}$ arm with the expanding bubble (Bubble A in Westmoquette et al. 2008 and Filament 6 in Hunter et al. 1993). The western $\mathrm{H} \alpha$ arm is morphologically very different from the other filaments in the galaxy and given the confluence of interesting $\mathrm{H} \alpha, \mathrm{HI}$, and radio continuum features 
in this region, it would be surprising if they were not related.

The extended radio halo around NGC 1569 may not be unique to this galaxy. In general, dwarf galaxies have very shallow potential wells, so any significant star formation episodes will tend out drive material out of their potential wells. This mechanism has been postulated as a way to enrich the intergalactic medium (Mac Low \& Ferrara 1999), but it could also be a mechanism to magnetize the intergalactic medium (Kronberg et al. 1999; Völk \& Atoyan 2000; Bertone et al. 2006). If these halos are like the radio continuum halo of NGC 1569, then they will be dominated by synchrotron emission. Therefore, the best wavelength regime to search for them is the low frequency radio regime with telescopes like LOFAR (Klein 2008). The detection of such halos in galaxies other than NGC 1569 would provide important clues about the magnetization of the universe and the escape of cosmic rays from the halos of galaxies.

\section{Summary and Conclusions}

NGC 1569 provides a crucial test of the importance of magnetic fields in starbursting dwarf galaxies. We present the first observations to date of the polarized radio continuum emission at $20 \mathrm{~cm}, 13 \mathrm{~cm}, 6 \mathrm{~cm}$, and $3 \mathrm{~cm}$ from the post-starburst dwarf irregular NGC 1569 . We detect radio continuum emission from NGC 1569 at all four wavelengths. Our observations are in agreement with previous high-resolution radio continuum observations of NGC 1569 Seaquist \& Bignell 1976; Condon 1983; Klein \& Gräve 1986; Israel \& de Bruyn 1988; Lisenfeld et al. 2004). We confirm the discovery of an extended radio continuum halo, which was first imaged by Condon (1983) and discussed by Israel \& de Bruyn (1988), and for the first time clearly image the western arm at wavelengths shorter than $20 \mathrm{~cm}$. In general, the spectral indices derived for this galaxy get steeper as one moves along the minor axis of the galaxy away from the disk. However, there are two filamentary regions of flat spectral indices extending to the edge of the map. The spectral index trends shown here agree with those found by Lisenfeld et al. (2004) supporting their theory that there is a convective wind at work in the halo of this galaxy.

We detect strong polarized emission at all four frequencies. The polarized emission at 6 and $3 \mathrm{~cm}$ is spread over most of the galaxy, while the polarized emission at $20 \mathrm{~cm}$ is much spottier, and there are only a few small patches of polarized emission at $13 \mathrm{~cm}$. The polarized emission at $20 \mathrm{~cm}, 6 \mathrm{~cm}$ and $3 \mathrm{~cm}$ is associated with regions identified as bubbles from $\mathrm{H} \alpha$ observations. There is little polarization in the central region of NGC 1569, where the ISM is very turbulent and the largest thermal electron column density can be found. The $\mathrm{H} \alpha$ filaments in the halo also depolarize the emission. 
In order to determine the magnetic field strength, we estimate the thermal contribution to the radio continuum emission using a flux-calibrated $\mathrm{H} \alpha$ image. We find that the thermal contribution peaks at $40-50 \%$ in the center of the galaxy and falls off quickly. The halo is dominated by synchrotron emission. The total magnetic field strength in the central regions is $38 \mu \mathrm{G}$ and approximately $10-15 \mu \mathrm{G}$ in the halo. The field is largely random near the center; the uniform field is strongest in the halo. The magnetic field structure of NGC 1569 is shaped by the outflow of gas from this galaxy drawing field loops out of the disk. We show that the magnetic field structure of NGC 1569 is closely aligned with the H $\alpha$ bubbles.

We find that the magnetic pressure is the same order of magnitude as other components of the pressure in the ISM, but is generally less than the pressures of other components in similar regions by a factor of a few. Therefore, the magnetic field does not dominate the ISM of NGC 1569, although it clearly plays an important role.

The close correspondence between the $\mathrm{H} \alpha$ bubbles and the magnetic field structure suggests that the interstellar medium of NGC 1569 contains magnetic bubbles similar to those modelled by Ferriere et al. (1991). The high supernova rate and strong shear in the galaxy compared to the Milky Way suggests that these bubbles might combine to give a supernova/superbubble driven dynamo like that of Ferrière \& Schmitt (2000) and Gressel et al. (2008). However, detailed modeling is necessary to say definitely whether or not this type of dynamo is at work in NGC 1569.

Finally, we compare our data to observations of the ISM of NGC 1569 at other wavelengths. The turbulent $\mathrm{H} \alpha$ kinematics of the central portion of the galaxy are reflected in a primarily random field, while the outflow kinematics in the halo are reflected by a uniform field. The magnetic field in the $\mathrm{H} \alpha$ arm may be increasing the width of this feature by providing an additional source of pressure as well as channeling hot gas out of the plane. We suggest that the infall of the western HI arm into the disk of NGC 1569 and the outward expansion of the bubble associated with the western $\mathrm{H} \alpha$ arm compressed the ISM in this region and produced the strong uniform magnetic field seen here. In short, our observations reveal that the magnetic field of NGC 1569 is shaped by the outflow of gas from the galaxy and its interaction history and that the magnetic field may be playing an important role in directing the outflow of gas from the galaxy.

Radio continuum halos generated by the outflow of gas from galaxy may be common in dwarf starburst galaxies. These halos provide a mechanism for magnetizing the intergalactic medium (Kronberg et al. 1999; Völk \& Atoyan 2000; Bertone et al. 2006). Surveys of edge-on dwarf starbursts with future low-frequency radio telescopes like LOFAR will help constrain the number of dwarf starbursts with synchrotron-dominated radio halos and provide important clues about the magnetization of the universe and the escape of cosmic rays from galaxy 
halos.

The authors would like to thank Deidre Hunter and Jürgen Ott for providing us with the $\mathrm{H} \alpha$ and X-ray images of NGC 1569 used in this paper. We also thank Rainer Beck, Crystal Brogan, Ger de Bruyn, Krzysztof Chẏzy, Bill Cotton, Bryan Gaensler, Jay Gallagher, Miller Goss, Dan McCammon, Jürgen Ott, and Snežana Stanimirović for helpful conversations. Finally, we would also like to acknowledge the extremely helpful comments made by the anonymous referee. A.A.K. was supported by an NSF Graduate Fellowship, a Wisconsin Space Grant Graduate Fellowship, and a GBT Student Support award during portions of this work. S.M. acknowledges the Deutsche Forschungsgemeinschaft for the award of a fellowship of the Graduiertenkolleg "The Magellanic System, Galaxy Interaction, and the Evolution of Dwarf Galaxies", support under grant SFB 494, and the hospitality of the Department of Astronomy at University of Wisconsin-Madison for her visit in February 2007. E.G.Z., J.E., and E.M.W. would like to acknowledge the support of NSF grants AST 0507367 (E.G.Z. and J.E.), PHY 0215581 (E.G.Z. and J.E.), and AST 0708002 (E.M.W.).

\section{A. Deriving the Geometric Corrections}

We assume that the geometry of NGC 1569 can be approximated by an infinite cylinder with the vector normal to the galaxy disk inclined from the line of sight by $i$ degrees $\left(i=0^{\circ}\right.$ is face-on, $i=90^{\circ}$ is edge-on) and rotated on the sky by a position angle (measured from north and increasing counterclockwise, i.e, towards the east) PAg. The geometry for NGC 1569 and the relevant variables are shown in Figures 13 and 14. Below we derive the relevant quantities needed for our calculation of the magnetic field strength.

\section{A.1. Deriving the Line of Sight Distance}

The line-of-sight length $(l)$ through an inclined (infinite) cylinder is

$$
l=\frac{2\left(R^{2}-d^{2}\right)^{1 / 2}}{\sin i}
$$

where $d$ is the distance from the central axis of the cylinder, $R$ is the radius of the cylinder, and $i$ is the inclination from the line of sight. If the cylinder is rotated about its minor axis in the plane of the sky, where the angle between the RA axis and the disk of the galaxy (minor axis of cylinder) is $\beta, d$ becomes

$$
d=D \frac{\pi}{180}\left[\left(\mathrm{RA}_{\mathrm{pix}}-\mathrm{RA}_{0}\right) \cos \left(\mathrm{Dec}_{0}\right) \cos \beta-\left(\mathrm{Dec}_{\mathrm{pix}}-\mathrm{Dec}_{0}\right) \sin \beta\right]
$$


where $D$ is the distance to the galaxy, $\left(\mathrm{RA}_{\mathrm{pix}}, \mathrm{Dec}_{\mathrm{pix}}\right)$ are the coordinates of a particular pixel, and $\left(\mathrm{RA}_{0}, \mathrm{Dec}_{0}\right)$ are the coordinates of the center of the galaxy. The variable $\beta$ is defined as positive for counterclockwise rotations and negative for clockwise rotations and is $90+\mathrm{PA}_{\mathrm{g}}$ for $\mathrm{PA}_{\mathrm{g}}<0$ and $\mathrm{PA}_{\mathrm{g}}-90$ for $\mathrm{PA}_{\mathrm{g}}>0$. If $\beta$ is $0^{\circ}$, then $d$ is just

$$
d=D \frac{\pi}{180}\left(\mathrm{RA}_{\mathrm{pix}}-\mathrm{RA}_{0}\right) \cos \left(\mathrm{Dec}_{0}\right)
$$

\section{A.2. Deriving $c_{4}$ for our geometry.}

To deal with the position angle of the galaxy, we correct the observed polarization vector position angles $\left(\mathrm{PA}_{\mathrm{B}, \mathrm{O}}\right)$, which are on the usual position angle system where $\mathrm{PA}_{\mathrm{g}}=0^{\circ}$ is north, $\mathrm{PA}_{\mathrm{g}}=90^{\circ}$ is east, and $\mathrm{PA}_{\mathrm{g}}=-90^{\circ}$ is west, so that they are centered on a coordinate system where a position angle of $0^{\circ}$ is along the axis of the cylinder and a position angle of $90^{\circ}$ is pointing toward the left (eastward) and a position angle of $-90^{\circ}$ is pointing toward the right (westward). For a negative position angle, the correction is

$$
\mathrm{PA}_{\mathrm{B}}=-\left(90^{\circ}+\mathrm{PA}_{\mathrm{g}}\right)+\mathrm{PA}_{\mathrm{B}, \mathrm{O}}
$$

and for a positive position angle

$$
\mathrm{PA}_{\mathrm{B}}=\left(90^{\circ}-\mathrm{PA}_{\mathrm{g}}\right)+\mathrm{PA}_{\mathrm{B}, \mathrm{O}}
$$

We rotate any resulting $\mathrm{PA}_{\mathrm{B}}$ outside the range of $[90,-90]$ by $180^{\circ}$.

In the frame of the cylinder, there are two components to the observed magnetic field vector $\left(B_{\perp}\right): B_{x}$ and $B_{z, \perp}$. The $B_{x}$ components are not affected by the inclination of the galaxy because they are perpendicular to the inclination axis. In the $z$ direction, however, we only see the field strength $B_{z}$ projected onto the plane of the sky $\left(B_{z, \perp}\right)$. The observed magnetic field strength in the $z$ direction is

$$
B_{z, \perp}=B_{z} \sin i
$$

where $B_{z}$ is the magnetic field strength in the $z$ direction and $i$ is the inclination. Now the observed magnetic field strength $\left(B_{\perp}\right)$ is also equal to

$$
B_{z, \perp}=B_{\perp} \cos \left(\mathrm{PA}_{\mathrm{B}}\right)
$$

Therefore,

$$
B_{z}=B_{\perp} \cos \left(\mathrm{PA}_{\mathrm{B}}\right) / \sin i .
$$


Finally, the total magnetic field $\left(B_{t}\right)$ is

$$
\begin{aligned}
B_{t} & =\left(B_{x}^{2}+B_{z}^{2}\right)^{1 / 2} \\
& =\left[\left(B_{\perp} \sin \left(\mathrm{PA}_{\mathrm{B}}\right)\right)^{2}+\left(\frac{B_{\perp} \cos \left(\mathrm{PA}_{\mathrm{B}}\right)}{\sin i}\right)^{2}\right]^{1 / 2} \\
& =B_{\perp}\left[\left(\sin \left(\mathrm{PA}_{\mathrm{B}}\right)\right)^{2}+\left(\frac{\left.\cos (\mathrm{PA})_{\mathrm{B}}\right)}{\sin i}\right)^{2}\right]^{1 / 2}
\end{aligned}
$$

Beck \& Krause 2005) define $c_{4}$ as

$$
c_{4}=\left(\frac{B_{\perp}}{B_{t}}\right)^{(1-\alpha)}
$$

so our $c_{4}$ is then

$$
c_{4}=\left[\left(\sin \left(\mathrm{PA}_{\mathrm{B}}\right)\right)^{2}+\left(\frac{\cos (\mathrm{PA})}{\sin i}\right)^{2}\right]^{-(1-\alpha) / 2}
$$

for a completely regular field.

\section{REFERENCES}

Aloisi, A., Clampin, M., Diolaiti, E., Greggio, L., Leitherer, C., Nota, A., Origlia, L., Parmeggiani, G., \& Tosi, M. 2001, AJ, 121, 1425

Angeretti, L., Tosi, M., Greggio, L., Sabbi, E., Aloisi, A., \& Leitherer, C. 2005, AJ, 129, 2203

Beck, R. 2007, A\&A, 470, 539

Beck, R., Brandenburg, A., Moss, D., Shukurov, A., \& Sokoloff, D. 1996, ARA\&A, 34, 155

Beck, R., Carilli, C. L., Holdaway, M. A., \& Klein, U. 1994, A\&A, 292, 409

Beck, R., \& Krause, M. 2005, Astronomische Nachrichten, 326, 414

Beck, R., Shukurov, A., Sokoloff, D., \& Wielebinski, R. 2003, A\&A, 411, 99

Bertone, S., Vogt, C., \& Enßlin, T. 2006, MNRAS, 370, 319

Breitschwerdt, D., McKenzie, J. F., \& Voelk, H. J. 1991, A\&A, 245, 79

-. 1993, A\&A, 269, 54 
Brouw, W. N. 1996, in Astrophysics and Space Science Library, Vol. 207, The Westerbork Observatory, Continuing Adventure in, ed. E. Raimond \& R. Genee, 53-74

Burn, B. J. 1966, MNRAS, 133, 67

Caplan, J., \& Deharveng, L. 1986, A\&A, 155, 297

Carilli, C. L., Holdaway, M. A., Ho, P. T. P., \& de Pree, C. G. 1992, ApJ, 399, L59

Cattaneo, F., \& Hughes, D. W. 2009, MNRAS, 395, L48

Chyży, K. T. 2008, A\&A, 482, 755

Chyży, K. T., Soida, M., Bomans, D. J., Vollmer, B., Balkowski, C., Beck, R., \& Urbanik, M. 2006, A\&A, 447, 465

Condon, J. J. 1983, ApJS, 53, 459

-. 1992, ARA\&A, 30, 575

Cooper, J. L., Bicknell, G. V., Sutherland, R. S., \& Bland-Hawthorn, J. 2008, ApJ, 674, 157

Dahlem, M., Aalto, S., Klein, U., Booth, R., Mebold, U., Wielebinski, R., \& Lesch, H. 1990, A\&A, 240, 237

Dahlem, M., Petr, M. G., Lehnert, M. D., Heckman, T. M., \& Ehle, M. 1997, A\&A, 320, 731

de Avillez, M. A., \& Breitschwerdt, D. 2005, A\&A, 436, 585

de Vaucouleurs, G., de Vaucouleurs, A., \& Pence, W. 1974, ApJ, 194, L119

della Ceca, R., Griffiths, R. E., Heckman, T. M., \& MacKenty, J. W. 1996, ApJ, 469, 662

Devost, D., Roy, J.-R., \& Drissen, L. 1997, ApJ, 482, 765

Emerson, D. T., \& Gräve, R. 1988, A\&A, 190, 353

Emerson, D. T., Klein, U., \& Haslam, C. G. T. 1979, A\&A, 76, 92

Everett, J. E., Schiller, Q. G., \& Zweibel, E. G. 2009, ArXiv e-prints

Everett, J. E., Zweibel, E. G., Benjamin, R. A., McCammon, D., Rocks, L., \& Gallagher, III, J. S. 2008, ApJ, 674, 258

Ferrière, K., \& Schmitt, D. 2000, A\&A, 358, 125 
Ferrière, K. M. 2001, Reviews of Modern Physics, 73, 1031

Ferriere, K. M., Mac Low, M., \& Zweibel, E. G. 1991, ApJ, 375, 239

Gardner, F. F., \& Whiteoak, J. B. 1966, ARA\&A, 4, 245

Ginzburg, V. L., \& Syrovatskii, S. I. 1965, ARA\&A, 3, 297

Golla, G., \& Hummel, E. 1994, A\&A, 284, 777

Greggio, L., Tosi, M., Clampin, M., de Marchi, G., Leitherer, C., Nota, A., \& Sirianni, M. 1998, ApJ, 504, 725

Greisen, E., ed. 2006, AIPS COOKBOOK (NRAO)

Gressel, O., Elstner, D., Ziegler, U., \& Rüdiger, G. 2008, A\&A, 486, L35

Grocholski, A. J., Aloisi, A., van der Marel, R. P., Mack, J., Annibali, F., Angeretti, L., Greggio, L., Held, E. V., Romano, D., Sirianni, M., \& Tosi, M. 2008, ApJ, 686, L79

Haslam, C. G. T. 1974, A\&AS, 15, 333

Heckman, T. M., Dahlem, M., Lehnert, M. D., Fabbiano, G., Gilmore, D., \& Waller, W. H. 1995, ApJ, 448, 98

Heesen, V., Beck, R., Krause, M., \& Dettmar, R.-J. 2009, A\&A, 494, 563

Heiles, C. 1996, in Astronomical Society of the Pacific Conference Series, Vol. 97, Polarimetry of the Interstellar Medium, ed. W. G. Roberge \& D. C. B. Whittet, 457-474

Hillas, A. M. 2006, ArXiv Astrophysics e-prints

Hodge, P. W. 1974, ApJ, 191, L21

Hunt, L. K., Dyer, K. K., Thuan, T. X., \& Ulvestad, J. S. 2004, ApJ, 606, 853

Hunter, D. A., \& Elmegreen, B. G. 2004, AJ, 128, 2170

Hunter, D. A., \& Gallagher, III, J. S. 1997, ApJ, 475, 65

Hunter, D. A., Hawley, W. N., \& Gallagher, III, J. S. 1993, AJ, 106, 1797

Israel, F. P., \& de Bruyn, A. G. 1988, A\&A, 198, 109

Johnston-Hollitt, M., Hollitt, C. P., \& Ekers, R. D. 2004, in The Magnetized Interstellar Medium, ed. B. Uyaniker, W. Reich, \& R. Wielebinski, 13-18 
Klein, U. 2008, in IAU Symposium, Vol. 255, IAU Symposium, 167-172

Klein, U., \& Gräve, R. 1986, A\&A, 161, 155

Klein, U., Wielebinski, R., \& Morsi, H. W. 1988, A\&A, 190, 41

Kronberg, P. P., Lesch, H., \& Hopp, U. 1999, ApJ, 511, 56

Kulsrud, R. M., \& Zweibel, E. G. 2008, Reports on Progress in Physics, 71, 046901

Lisenfeld, U., Wilding, T. W., Pooley, G. G., \& Alexander, P. 2004, MNRAS, 349, 1335

Mac Low, M.-M., \& Ferrara, A. 1999, ApJ, 513, 142

Martin, C. L. 1997, ApJ, 491, 561

-. 1998, ApJ, 506, 222

-. 1999, ApJ, 513, 156

Martin, C. L., \& Kennicutt, Jr., R. C. 1997, ApJ, 483, 698

Martin, C. L., Kobulnicky, H. A., \& Heckman, T. M. 2002, ApJ, 574, 663

Mineshige, S., Shibata, K., \& Shapiro, P. R. 1993, ApJ, 409, 663

Mühle, S., Klein, U., Wilcots, E. M., \& Hüttemeister, S. 2005, AJ, 130, 524

-. 2006, AJ, 132, 443

Otmianowska-Mazur, K., Soida, M., Kulesza-Żydzik, B., Hanasz, M., \& Kowal, G. 2009, ApJ, 693, 1

Ott, J., Walter, F., \& Brinks, E. 2005a, MNRAS, 358, 1423

-. 2005b, MNRAS, 358, 1453

Pacholczyk, A. G. 1970, Radio astrophysics. Nonthermal processes in galactic and extragalactic sources (Series of Books in Astronomy and Astrophysics, San Francisco: Freeman, 1970)

Parker, E. N. 1992, ApJ, 401, 137

Relaño, M., Lisenfeld, U., Vilchez, J. M., \& Battaner, E. 2006, A\&A, 452, 413

Reuter, H.-P., \& Klein, U. 1996, A\&A, 313, 768 
Reuter, H.-P., Klein, U., Lesch, H., Wielebinski, R., \& Kronberg, P. P. 1994, A\&A, 282, 724

Robishaw, T., Quataert, E., \& Heiles, C. 2008, ApJ, 680, 981

Schwartz, C. M., \& Martin, C. L. 2004, ApJ, 610, 201

Seaquist, E. R., \& Bignell, R. C. 1976, A\&A, 48, 421

Simmons, J. F. L., \& Stewart, B. G. 1985, A\&A, 142, 100

Sokoloff, D. D., Bykov, A. A., Shukurov, A., Berkhuijsen, E. M., Beck, R., \& Poezd, A. D. 1998, MNRAS, 299, 189

Stil, J. M., \& Israel, F. P. 2002, A\&A, 392, 473

Tabatabaei, F. S., Krause, M., Fletcher, A., \& Beck, R. 2008, A\&A, 490, 1005

Tenorio-Tagle, G., Muñoz-Tuñón, C., Pérez, E., Silich, S., \& Telles, E. 2006, ApJ, 643, 186

Thompson, T. A., Quataert, E., Waxman, E., Murray, N., \& Martin, C. L. 2006, ApJ, 645, 186

Tody, D. 1986, in Presented at the Society of Photo-Optical Instrumentation Engineers (SPIE) Conference, Vol. 627, Society of Photo-Optical Instrumentation Engineers (SPIE) Conference Series, ed. D. L. Crawford, 733-+

Tody, D. 1993, in Astronomical Society of the Pacific Conference Series, Vol. 52, Astronomical Data Analysis Software and Systems II, ed. R. J. Hanisch, R. J. V. Brissenden, \& J. Barnes, 173-+

Tomisaka, K. 1990, ApJ, 361, L5

Tomita, A., Ohta, K., \& Saito, M. 1994, PASJ, 46, 335

Ulvestad, J. S., P. R. A., \& Taylor, G. B., e. 2007, The Very Large Array Observational Status Summary, National Radio Astronomy Observatory

Vaillancourt, J. E. 2006, PASP, 118, 1340

Vallenari, A., \& Bomans, D. J. 1996, A\&A, 313, 713

Völk, H. J., \& Atoyan, A. M. 2000, ApJ, 541, 88

Waller, W. H. 1991, ApJ, 370, 144 
Westmoquette, M. S., Exter, K. M., Smith, L. J., \& Gallagher, J. S. 2007a, MNRAS, 381, 894

Westmoquette, M. S., Smith, L. J., \& Gallagher, J. S. 2008, MNRAS, 383, 864

Westmoquette, M. S., Smith, L. J., Gallagher, J. S., \& Exter, K. M. 2007b, MNRAS, 381, 913 


\section{Table 1. Summary of WSRT $20 \mathrm{~cm}$ Observations}

\begin{tabular}{rcc}
\hline \hline & $5-6$ Nov & $6-7$ Nov \\
& 2000 & 2000 \\
\hline Array Configuration & $36 \mathrm{~m}$ & $72 \mathrm{~m}$ \\
IF1 (GHz) & 1.345 & 1.345 \\
IF2 (GHz) & 1.355 & 1.355 \\
IF3 (GHz) & 1.365 & 1.365 \\
IF4 (GHz) & 1.375 & 1.375 \\
IF5 (GHz) & 1.385 & 1.385 \\
IF6 (GHz) & 1.395 & 1.395 \\
IF7 (GHz) & 1.405 & 1.405 \\
IF8 (GHz) & 1.415 & 1.415 \\
Bandwidth per IF (MHZ) & 10 & 10 \\
Flux Calibrator & $0137+331$ & $1331+305$ \\
Instrumental & $0137+331$ & $0542+498$ \\
Polarization & & \\
Absolute & $2253+161$ & $1331+305$ \\
Polarization Angle & & \\
\hline
\end{tabular}

Table 2. Final Images

\begin{tabular}{|c|c|c|c|c|c|c|c|c|}
\hline Data Name & $\begin{array}{c}\text { Frequency } \\
\text { GHz }\end{array}$ & Weighting & $\begin{array}{l}\text { Beam } \\
\quad \prime\end{array}$ & $\begin{array}{c}\text { PA } \\
\circ\end{array}$ & $\begin{array}{c}\mathrm{LAS}^{\mathrm{a}} \\
\quad\end{array}$ & $\begin{array}{c}\sigma_{I} \\
\mu \mathrm{Jy}^{-1} \text { beam }^{-1}\end{array}$ & $\begin{array}{c}\sigma_{Q} \\
\mu \mathrm{Jy}_{\text {beam }^{-1}}\end{array}$ & $\begin{array}{c}\sigma_{U} \\
\mu \mathrm{Jy}_{\text {beam }^{-1}}\end{array}$ \\
\hline $20 \mathrm{~cm}$ & 1.38 & Natural $^{\mathrm{b}}$ & $12.88 \times 14.19$ & 6.5 & 21 & 16.5 & 12.6 & 13.2 \\
\hline $13 \mathrm{~cm}, 13^{\prime \prime}$ & 2.27 & uvrange $=(0.165,13)$ & $13.44 \times 12.96$ & -9.60 & 13 & 24.8 & 19.4 & 19.3 \\
\hline $6 \mathrm{~cm}$, robust $=0$ & 4.86 & Robust $=0$ & $4.42 \times 4.08$ & 89.77 & $\ldots \mathrm{c}$ & 8.5 & 7.6 & 7.6 \\
\hline $6 \mathrm{~cm}, 13^{\prime \prime}$ & 4.86 & uvrange $=(0.165,13)$ & $12.64 \times 11.66$ & -88.20 & $\ldots \mathrm{c}$ & 14.0 & 10.8 & 10.7 \\
\hline
\end{tabular}

${ }^{a}$ Largest Angular Scale

${ }^{\mathrm{b}}$ All fixed-moveable baselines selected.

${ }^{\mathrm{c}}$ This image is the result of combining VLA and Effelsberg observations, so it includes all spatial frequencies greater than the frequency of the synthesized beam. 
Table 3. Summary of WSRT $13 \mathrm{~cm}$ Observations

\begin{tabular}{rcc}
\hline \hline & $4-5 \mathrm{Dec}$ & $\begin{array}{c}18-19 \text { Jan } \\
2003\end{array}$ \\
& 2002 & $36 \mathrm{~m}$ \\
Array Configuration & $72 \mathrm{~m}$ & 2.20000 \\
IF1 (GHz) & 2.200000 & 2.218125 \\
IF2 (GHz) & 2.218125 & 236250 \\
IF3 (GHz) & 2.236250 & 2.2360 \\
IF4 (GHz) & 2.254375 & 2.254375 \\
IF5 (GHz) & 2.272500 & 2.272500 \\
IF6 (GHz) & 2.290625 & 2.290625 \\
IF7 (GHz) & 2.308750 & 2.308750 \\
IF8 (GHZ) & 2.326875 & 2.326875 \\
Bandwidth per IF (MHz) & 20 & 20 \\
Bandpass Calibrator & $1331+305$ & $1331+305$ \\
Flux Calibrator & $1331+305$ & $1331+305$ \\
Instrumental & $0834+555$ & $0834+555$ \\
Polarization & & \\
Absolute & $1331+305$ & $1331+305$ \\
Polarization Angle & & \\
R-L Angle & 66 & 66 \\
\hline
\end{tabular}

Table 4. Summary of VLA $6 \mathrm{~cm}$ and $3 \mathrm{~cm}$ Observations

\begin{tabular}{|c|c|c|c|c|c|c|c|c|}
\hline & $\begin{array}{l}11 \text { Aug. } \\
2000\end{array}$ & $\begin{array}{l}13 \text { Aug. } \\
2000\end{array}$ & $\begin{array}{l}17 \text { Aug. } \\
2000\end{array}$ & $\begin{array}{l}27 \text { Aug. } \\
2000\end{array}$ & $\begin{array}{l}19 \text { Sept. } \\
2000\end{array}$ & $\begin{array}{l}23 \text { Sept. } \\
2000\end{array}$ & $\begin{array}{l}9 \text { Aug. } \\
2001\end{array}$ & $\begin{array}{l}10 \text { Aug. } \\
2001\end{array}$ \\
\hline Program Number & AM643 & AM643 & AM643 & AM643 & AM643 & AM643 & AM694 & AM694 \\
\hline Array & D & $\mathrm{D}$ & D & D & $\mathrm{D}$ & D & C & C \\
\hline $\mathrm{IF} 1(\mathrm{GHz})$ & 8.4351 & 4.8851 & 8.4351 & 8.4351 & 8.4351 & 8.4351 & 4.8851 & 4.8851 \\
\hline $\mathrm{IF} 2(\mathrm{GHz})$ & 8.4851 & 4.8351 & 8.4851 & 8.4851 & 8.4851 & 8.4851 & 4.8351 & 4.8351 \\
\hline Bandwidth (MHz) & 50 & 50 & 50 & 50 & 50 & 50 & 50 & 50 \\
\hline Flux Calibrator & $0521+166$ & $\begin{array}{l}1331+305 \\
0521+166\end{array}$ & $0521+166$ & $1331+305$ & $\begin{array}{l}1331+305 \\
0521+166\end{array}$ & $\begin{array}{l}1331+305 \\
0521+166\end{array}$ & $0137+331$ & $0137+331$ \\
\hline Phase Calibrator & $0244+624$ & $0614+607$ & $0244+624$ & $0614+607$ & $\begin{array}{l}0244+624 \\
0614+607\end{array}$ & $\begin{array}{l}0244+624 \\
0614+607\end{array}$ & $\begin{array}{l}0614+607 \\
0217+738\end{array}$ & $\begin{array}{l}0614+607 \\
0217+738\end{array}$ \\
\hline $\begin{array}{l}\text { Instrumental } \\
\text { Polarization }\end{array}$ & $0244+624$ & $\begin{array}{l}0319+415 \\
0614+607\end{array}$ & $0244+624$ & $0614+607$ & $\begin{array}{l}0244+624 \\
0614+607\end{array}$ & $\begin{array}{l}0244+624 \\
0614+607\end{array}$ & $\begin{array}{l}0614+607 \\
0217+738\end{array}$ & $\begin{array}{l}0614+607 \\
0217+738\end{array}$ \\
\hline Absolute & $0521+166$ & $1331+305$ & $0521+166$ & $1331+305$ & $1331+305$ & $0521+166$ & $0521+166$ & $1331+305$ \\
\hline $\begin{array}{r}\text { Polarization Angle } \\
\text { R-L Angle }\end{array}$ & $-20^{\circ}$ & $66^{\circ}$ & $-20^{\circ}$ & $66^{\circ}$ & $66^{\circ}$ & $-22^{\circ}$ & $-24^{\circ}$ & $66^{\circ}$ \\
\hline
\end{tabular}

Table 5. Summary of Effelsberg 100-m Observations

\begin{tabular}{ccccccc}
\hline \hline $\begin{array}{c}\text { Frequency } \\
\text { GHz }\end{array}$ & $\begin{array}{c}\text { Bandwidth } \\
\text { MHz }\end{array}$ & Number of Feeds & $\begin{array}{c}\text { Beam } \\
\text { ( }\end{array}$ & $\begin{array}{c}\text { Map Size } \\
\text { ( }\end{array}$ & $\begin{array}{c}\text { Observing Time } \\
\text { hr }\end{array}$ & $\begin{array}{c}\text { Noise } \\
\text { mJy beam }^{-1}\end{array}$ \\
\hline .85 & 500 & 2 & $2.7 \times 2.5$ & $10 \times 10$ & 5 & 2.5 \\
10.45 & 300 & 4 & $1.1 \times 1.1$ & $12 \times 8$ & 26 & 0.5 \\
\hline
\end{tabular}


Table 6. Parameters of Previous Observations

\begin{tabular}{|c|c|c|c|c|c|}
\hline Paper & $\begin{array}{l}\text { Wavelength } \\
\mathrm{cm}\end{array}$ & $\begin{array}{l}\text { Frequency } \\
\text { GHz }\end{array}$ & $\begin{array}{l}\text { Resolution } \\
\prime \prime\end{array}$ & Largest Angular Scale & $\begin{array}{c}\text { Sensitivity } \\
\text { mJy beam }^{-1}\end{array}$ \\
\hline \multirow[t]{2}{*}{ Seaquist \& Bignell (1976) } & 11.1 & 2.7 & $7.7 \times 5.5$ & 4 & $0.71 \mathrm{mJy}$ beam $^{-1}$ \\
\hline & 3.7 & 8.1 & $3.0 \times 2.1$ & 1.3 & $0.23 \mathrm{mJy}^{\text {beam }^{-1}}$ \\
\hline \multirow[t]{2}{*}{ Condon (1983) } & 21.4 & 1.4 & $\sim 15-20$ & 15 & $0.04 \mathrm{mJy} \mathrm{beam}^{-1}$ \\
\hline & 21.4 & 1.4 & $5.9 \times 5.8$ & 2 & $0.06 \mathrm{mJy}$ beam $^{-1}$ \\
\hline Klein \& Gräve (1986) & 1.2 & 24.5 & 29.7 & $\ldots$ a & $1.7 \mathrm{mJy}$ beam $^{-1}$ \\
\hline \multirow[t]{3}{*}{ Israel \& de Bruyn (1988) } & 50 & 0.6 & $56 \times 62$ & 48 & $\ldots \mathrm{b}$ \\
\hline & 21.4 & 1.4 & $24 \times 27$ & 14 & $0.65 \mathrm{mJy}$ beam $^{-1}$ \\
\hline & 6 & 5.0 & $6.9 \times 7.6$ & 6 & $\ldots \mathrm{b}$ \\
\hline \multirow[t]{4}{*}{ Lisenfeld et al. (2004) } & 20 & 1.5 & 6 & 2 & $0.12 \mathrm{mJy} \mathrm{beam}^{-1}$ \\
\hline & 6.1 & 4.9 & 6 & 2 & $0.13 \mathrm{mJy}$ beam $^{-1}$ \\
\hline & 3.6 & 8.4 & 6 & 2 & $0.09 \mathrm{mJy}^{\text {beam }^{-1}}$ \\
\hline & 1.9 & 15.4 & 6 & 2 & $0.15 \mathrm{mJy}^{\text {beam }^{-1}}$ \\
\hline
\end{tabular}

${ }^{a}$ These are single-dish observations, which are sensitive to all spatial scales greater than their resolution.

${ }^{\mathrm{b}}$ Sensitivities not given.

Table 7. Pressures of Various Components of the ISM of NGC 1569

\begin{tabular}{|c|c|c|c|c|}
\hline Component & Input Values & Equation & Reference & $\begin{array}{c}\text { Pressure } \\
10^{5} \mathrm{~K} \mathrm{~cm}^{-3}\end{array}$ \\
\hline Magnetic Field (central) & $B=38 \mu \mathrm{G}$ & $B^{2} /(8 \pi k)$ & 1 & 4.2 \\
\hline Magnetic Field (halo) & $B=12 \mu \mathrm{G}$ & $B^{2} /(8 \pi k)$ & 1 & 0.41 \\
\hline Hot Gas (central) & $n_{e}=0.049 \mathrm{~cm}^{-3}, T=7.23 \times 10^{6} \mathrm{~K}$ & $2 n_{e} T$ & 2 & 7.2 \\
\hline Hot Gas (halo) & $n_{e}=0.028 \mathrm{~cm}^{-3}, T=7.23 \times 10^{6} \mathrm{~K}$ & $2 n_{e} T$ & 2 & 2.0 \\
\hline HII Regions & $n_{e} \sim 57 \mathrm{~cm}^{-3}, T=1.0 \times 10^{4} \mathrm{~K}$ & $2 n_{e} T$ & 3 & 11 \\
\hline Diffuse Ionized Gas (DIG) & equilibrium between hot gas and DIG & - & 4 & $1-6.3$ \\
\hline Warm Gas (turbulent, broad) & $n_{e} \sim 0.1 \mathrm{~cm}^{-3}, v_{t u r b}=150 \mathrm{~km} \mathrm{~s}^{-1}$ & $n_{e} m_{H} v_{\text {turb }}^{2} /(2 k)$ & 5 & 1.4 \\
\hline Warm Gas (turbulent, narrow) & $n_{e} \sim 57 \mathrm{~cm}^{-3}, v_{\text {turb }}=70 \mathrm{~km} \mathrm{~s}^{-1}$ & $n_{e} m_{H} v_{\text {turb }}^{2} /(2 k)$ & 3,5 & 170 \\
\hline Gravity & $v_{\text {rad }}=65.5 \mathrm{~km} \mathrm{~s}^{-1}, i=63^{\circ}, r=1.4 \mathrm{kpc}$ & $3\left(v_{r a d} / \sin i\right)^{4} /\left(20 \pi r^{2} G k\right)$ & 6 & 8.1 \\
\hline
\end{tabular}

References. - (1): This work, (2): Ott et al. (2005b), (3): Martin (1997), (4): Martin (1999), (5): Westmoquette et al. (2008), (6): Mühle et al. (2005) 
Table 8. Magnetic Field Strengths in Starburst Galaxies

\begin{tabular}{|c|c|c|c|c|}
\hline Galaxy & $\begin{array}{r}B_{t} \\
\mu \mathrm{G}\end{array}$ & $\begin{array}{c}B_{t, \text { center }} \\
\mu \mathrm{G}\end{array}$ & $\begin{array}{c}B_{t, \text { halo }} \\
\mu \mathrm{G}\end{array}$ & Reference \\
\hline M82 & 50 & $\ldots$ & $\ldots$ & 1 \\
\hline NGC 253 & $\ldots$ & 17 & 7 & 2 \\
\hline NGC 4666 & $\ldots$ & 14.4 & 7.1 & 3 \\
\hline NGC 1808 & $\ldots$ & 75 & $10-35$ & 4 \\
\hline NGC 1569 & $\ldots$ & 38 & $10-15$ & 5 \\
\hline
\end{tabular}

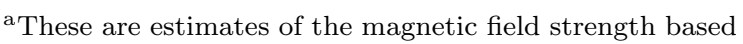
on equipartition between gravitational and magnetic pressures from Thompson et al. (2006).

References. - (1): Klein et al. (1988), (2): Beck et al. (1994), (3): Dahlem et al. (1997), (4): Dahlem et al. (1990),

(5): This work 


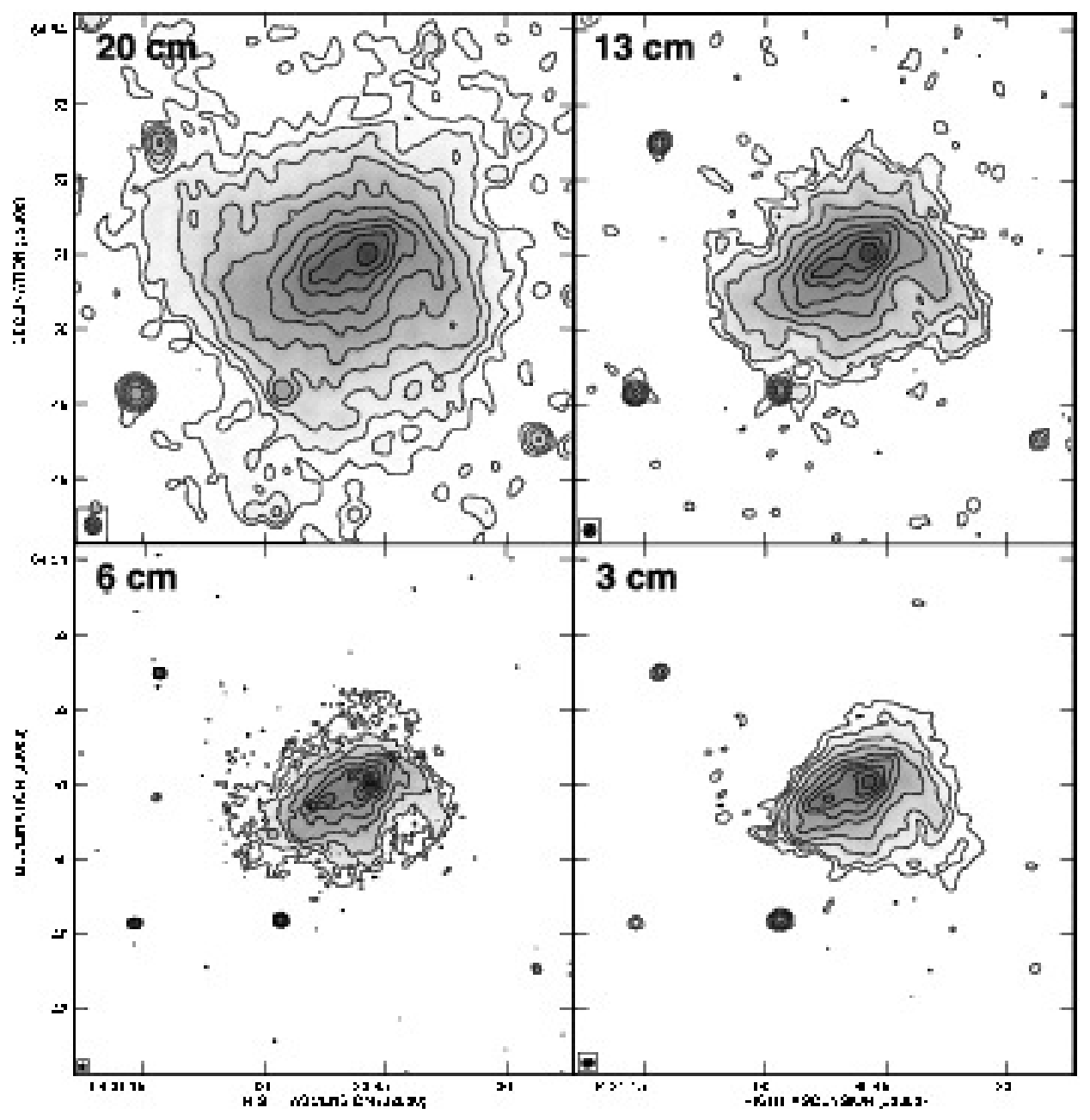

Fig. 1.- $20 \mathrm{~cm}, 13 \mathrm{~cm}, 6 \mathrm{~cm}$, and $3 \mathrm{~cm}$ total intensity, robust $=0$ weighted images. The contours start at $3 \sigma$ and increase by factors of 2 (see Table 2 for the noise levels for each image). The beam for each observation appears in the lower left corner. See Figure 2 for a more detailed view of the $6 \mathrm{~cm}$ and $3 \mathrm{~cm}$ emission. 


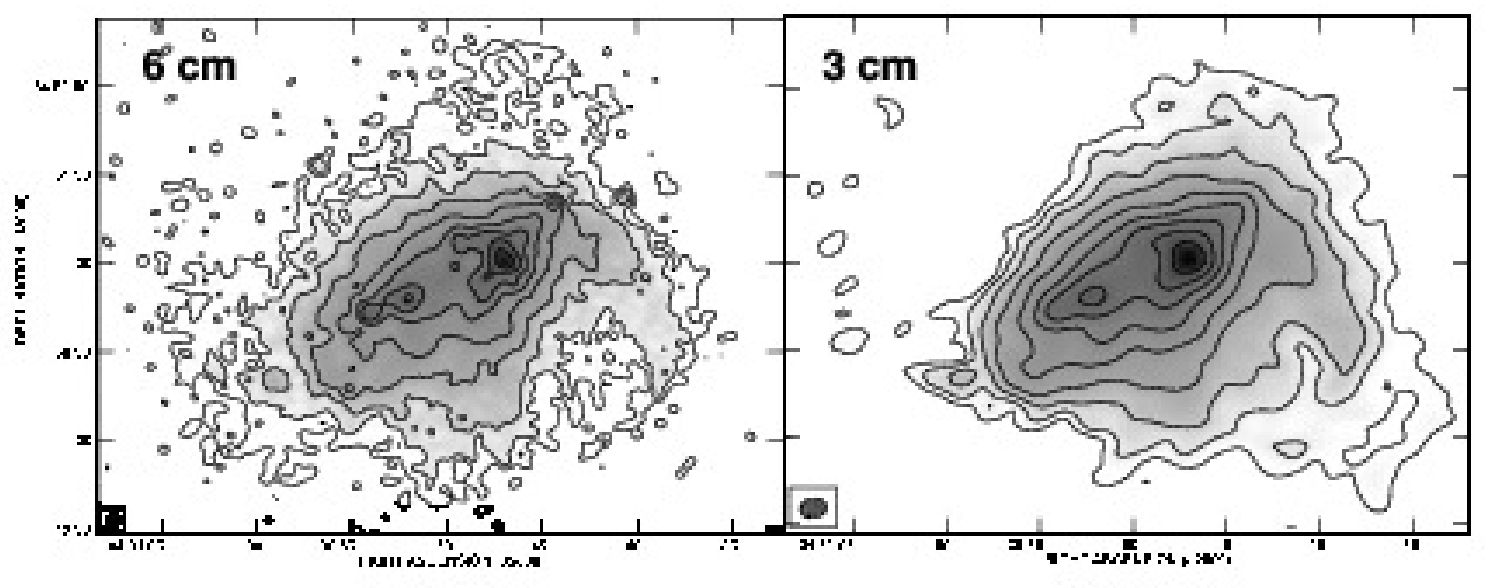

Fig. 2.- Zoomed-in version of the $6 \mathrm{~cm}$ and $3 \mathrm{~cm}$ total intensity, robust $=0$ weighted images. The first contour is at $3 \sigma$ and subsequent contours increase by factors of two (see Table 2 for the noise levels for various images). The beam appears in the lower left corner of each panel. 


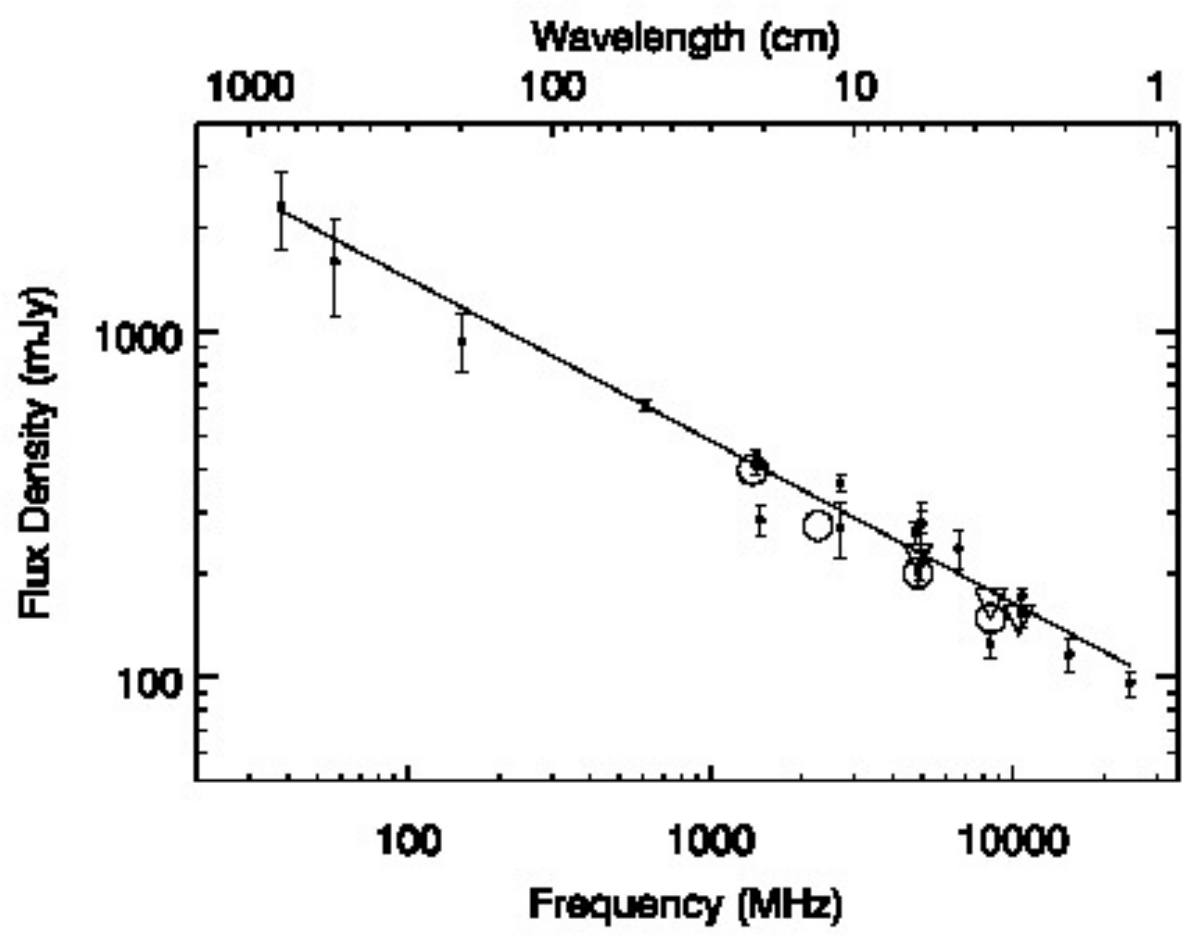

Fig. 3.- Integrated flux density spectrum for NGC 1569. The small dots are from the compilation of Lisenfeld et al. (2004) and open circles are the total flux in our final images. The error in the total flux of our final images is less than the size of the symbol. The open triangles are the total flux densities determined from our Effelsberg observations. The $8 \mathrm{GHz}$ flux was determined by scaling our $10 \mathrm{GHz}$ Effelsberg observations to $8 \mathrm{GHz}$ assuming a spectral index of 0.47 . The error bars show $1 \sigma$ errors. The line shows a power law with a slope of $\alpha=0.47$. 


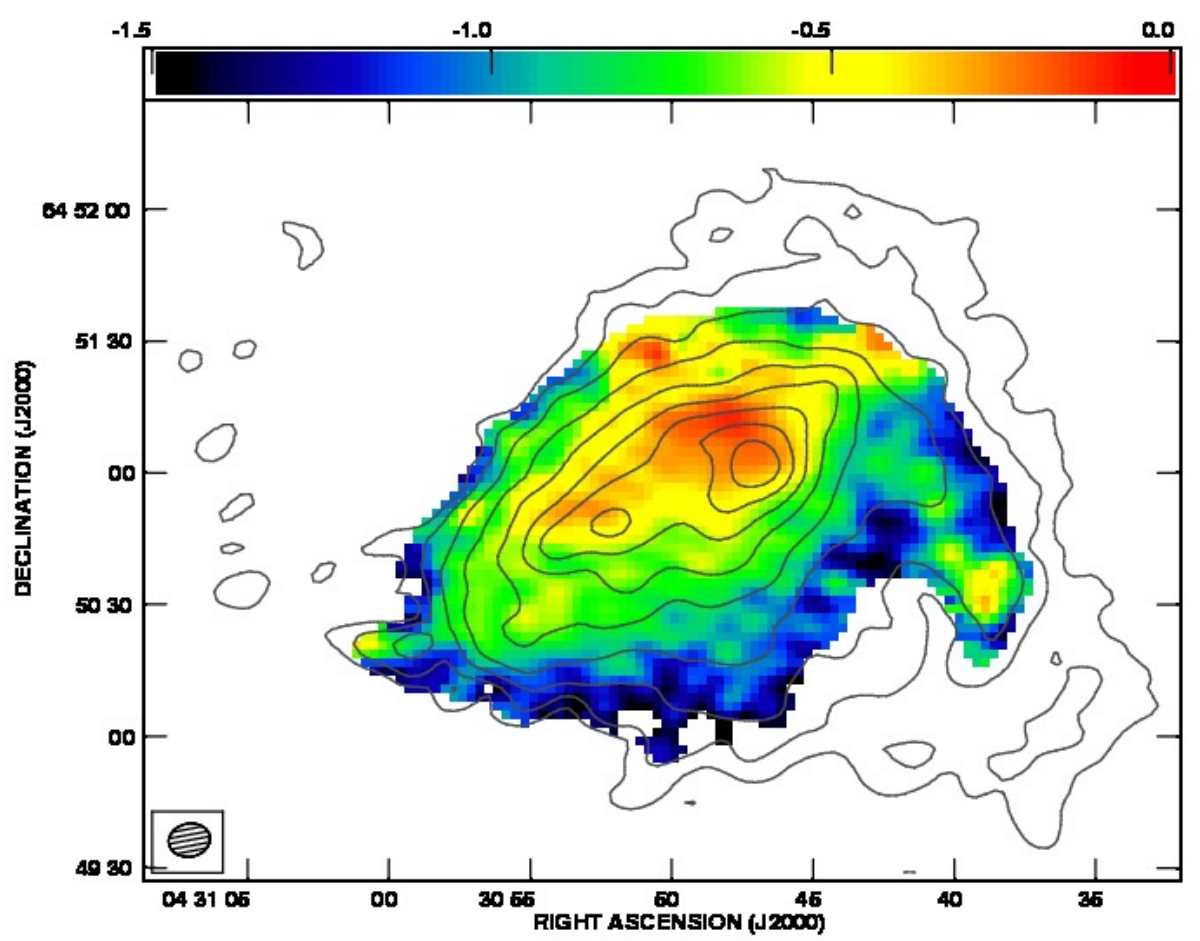

Fig. 4.- Spectral index map between $6 \mathrm{~cm}$ and $3 \mathrm{~cm}$ data. For this map, both data sets were imaged with the same $u-v$ range $(0.74$ to $20 \mathrm{k} \lambda$ ) and smoothed to the same beam size (9.34" by $7.54^{\prime \prime}$, shown in the lower left corner). Regions where the formal error on the spectral indices exceeded 0.2 were blanked. The contours show the $3 \mathrm{~cm}$, robust $=0$ image and start at the $3 \sigma$ noise level and increase by a factor of two for each subsequent contour. 


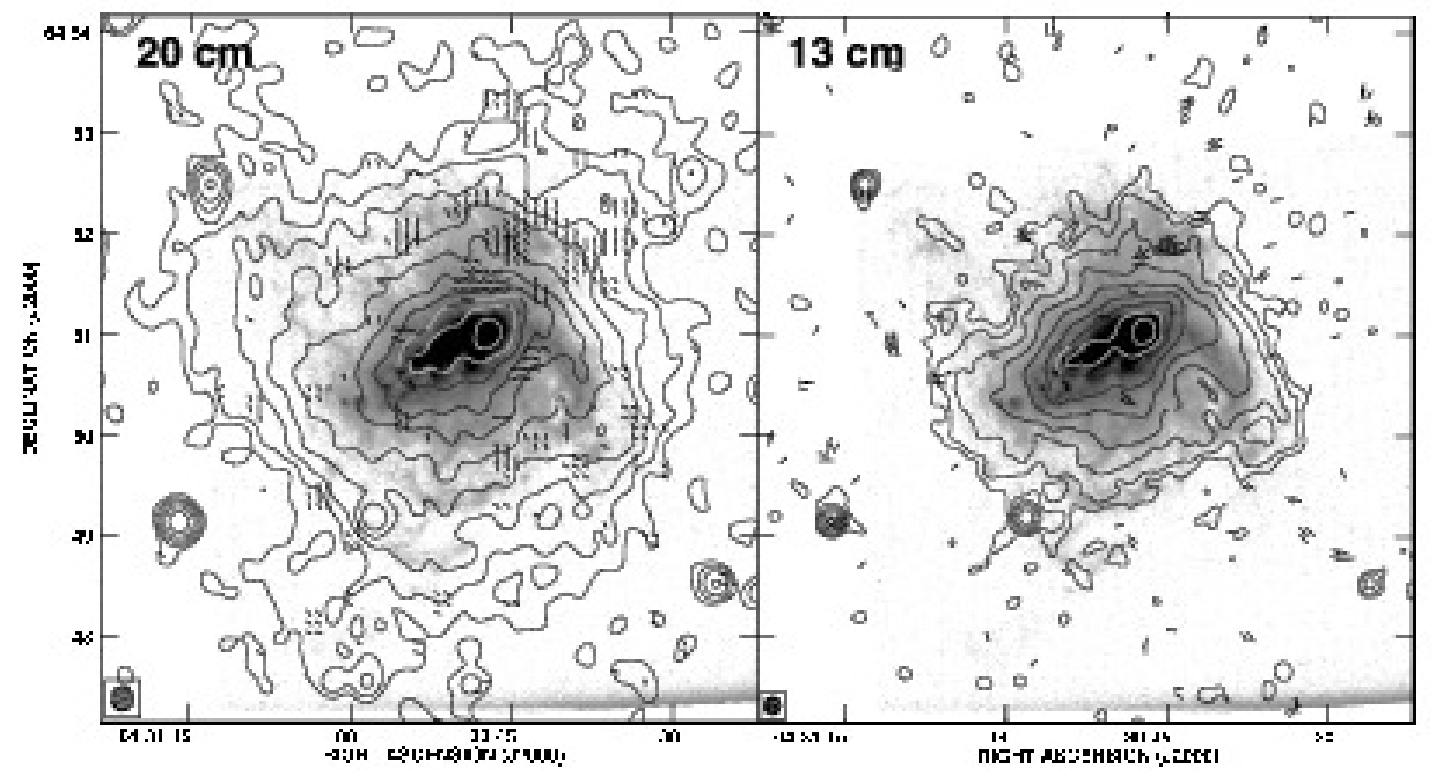

Fig. 5.- E-vectors showing the direction and distribution of the polarized intensity at $20 \mathrm{~cm}$ and $13 \mathrm{~cm}$ with contours showing the total intensity radio continuum emission at each wavelength (robust $=0$ weighting) and a grayscale image showing the distribution of the $\mathrm{H} \alpha$ emission. A polarization vector with a length of $5^{\prime \prime}$ has a polarized intensity of 51.1 $\mu \mathrm{Jy}_{\mathrm{beam}}{ }^{-1}$. The contour levels are for the robust $=0$ image in each case. They start at $3 \sigma$ and increase by a factor of 2 for subsequent contour (see Table 2 for the noise levels for each image). The central portion of NGC 1569 has been saturated in the grayscale image to better show the faint, extended emission. The beams are indicated the lower left hand corner of each panel. The line in the greyscale image roughly running from RA $04^{\mathrm{h}} 30^{\mathrm{m}} 45^{\mathrm{s}}$ to $04^{\mathrm{h}} 30^{\mathrm{m}} 22^{\mathrm{s}}$ at a declination of $64^{\circ} 47^{\prime}$ is due to the edge of the CCD. 


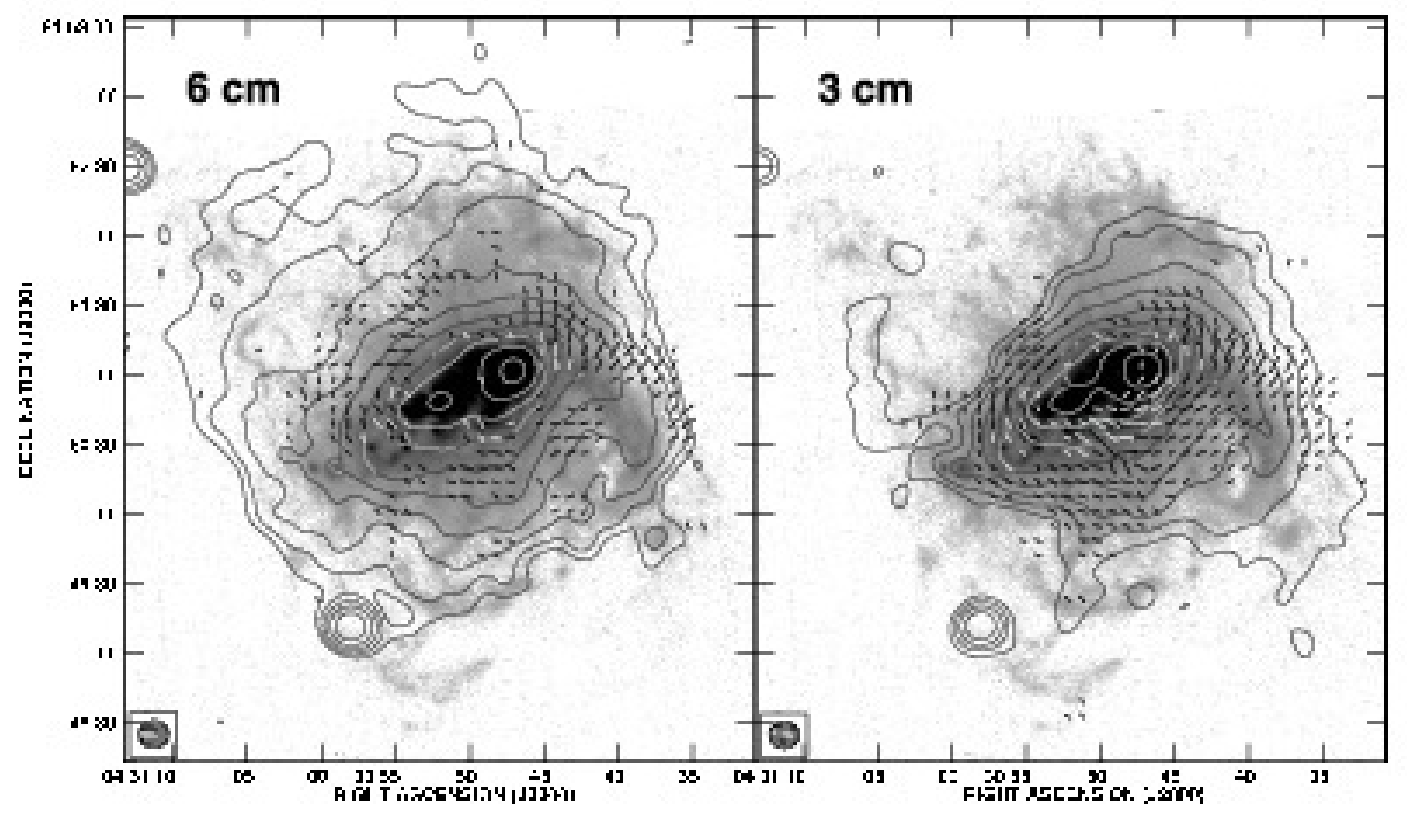

Fig. 6. - E-vectors showing the direction and distribution of polarized intensity at $3 \mathrm{~cm}$ and $6 \mathrm{~cm}$ with contours showing the total intensity radio continuum emission at each wavelength and a greyscale image showing the distribution of the $\mathrm{H} \alpha$ emission. We show the $6 \mathrm{~cm}$ and $3 \mathrm{~cm}$ data with $13^{\prime \prime}$ resolution because these were the data with the most polarized intensity. The contours start at $3 \sigma$ and increase by a factor of 2 for each subsequent contour. A polarization vector with a length of $1^{\prime \prime}$ has as polarized intensity of $12.8 \mu \mathrm{Jy} \mathrm{beam}^{-1}$. The beams are indicated in the lower left corner of each panel. 


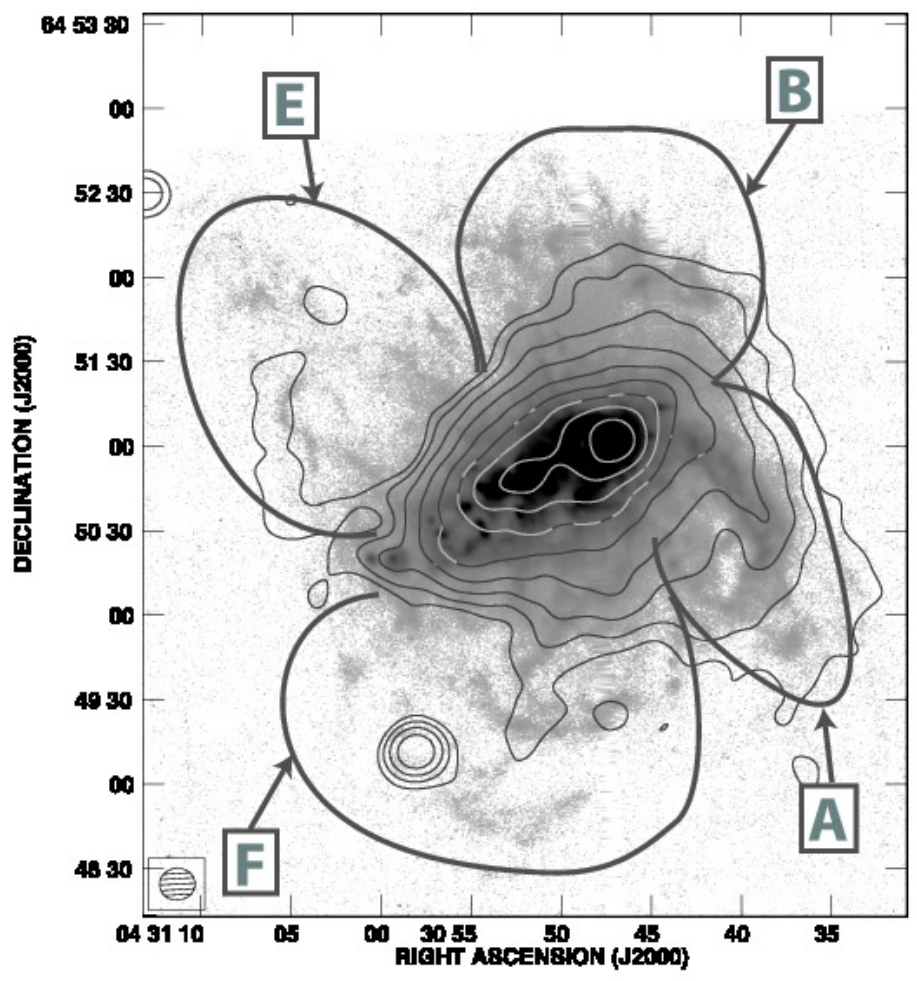

Fig. 7.- An H $\alpha$ image image from Hunter \& Elmegreen (2004) with outlines indicating the $\mathrm{H} \alpha$ bubbles from Martin (1998) and Westmoquette et al. (2008) and contours showing the $3 \mathrm{~cm}$ radio continuum emission with $13^{\prime \prime}$ resolution. The contours start at $3 \sigma$ and subsequent contours increase by factors of two. See Table 2 for the noise levels in each image and Figure 2 in Westmoquette et al. (2008) for the correspondence between this nomenclature and the nomenclature of Hunter et al. (1993). 


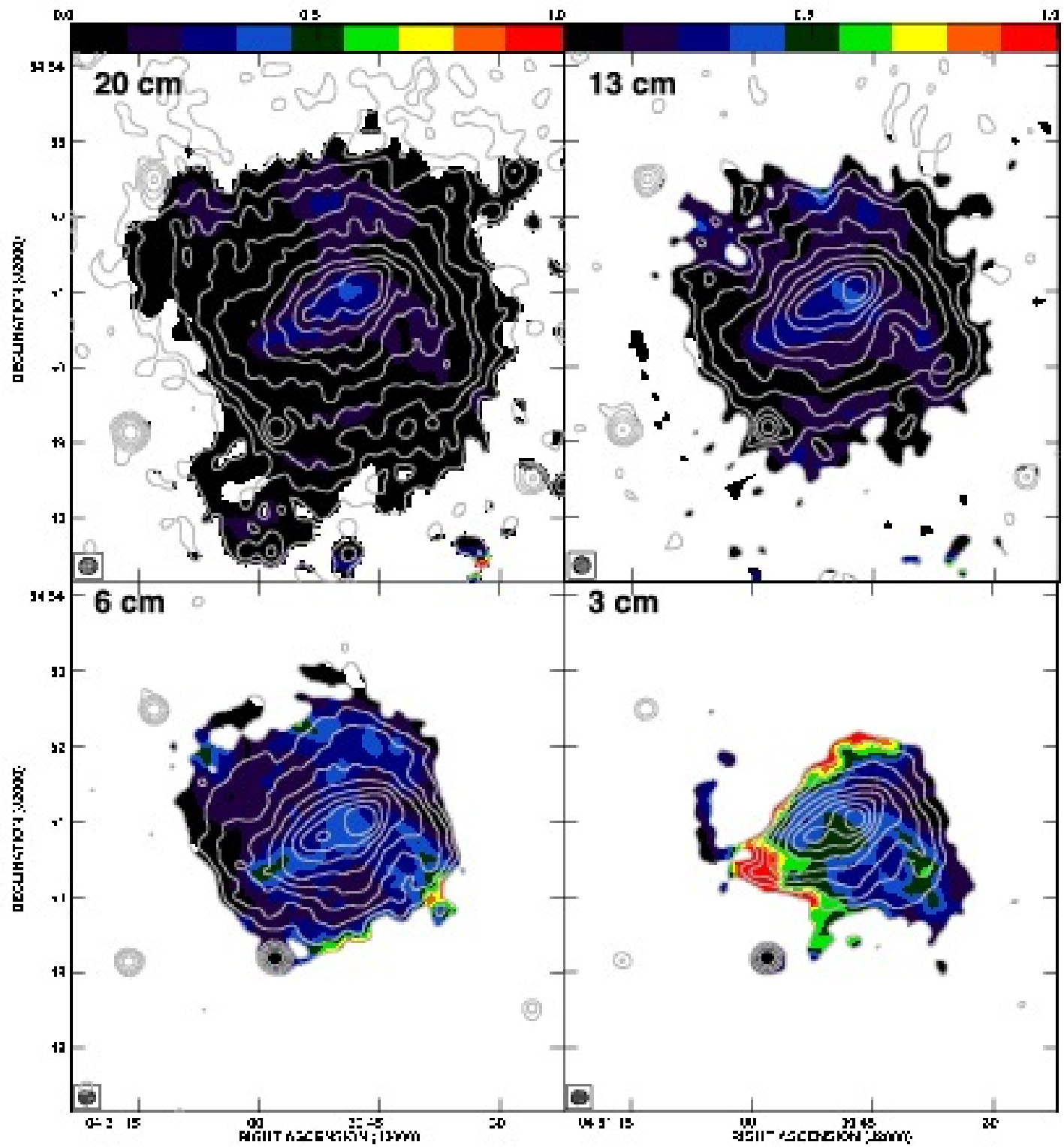

Fig. 8.- Fraction of the total emission at $20 \mathrm{~cm}, 13 \mathrm{~cm}, 6 \mathrm{~cm}$, and $3 \mathrm{~cm}$ that is thermal. The thermal emission was estimated using an $\mathrm{H} \alpha$ image of the galaxy (see $\S$ 4.1). Overlaid are the contours of total intensity at each wavelength with $13^{\prime \prime}$ resolution. The contours start at $3 \sigma$ and increase by a factor of 2 for each subsequent contour (see Table 2 for the noise levels for each of the images). The beam is shown in the lower left hand corner of each panel. The thermal fraction image at $20 \mathrm{~cm}$ does not extend to the boundaries of the $20 \mathrm{~cm}$ emission because the $20 \mathrm{~cm}$ radio continuum emission extends beyond the $\mathrm{H} \alpha$ image boundaries, particularly on the east and north sides of the galaxy. 

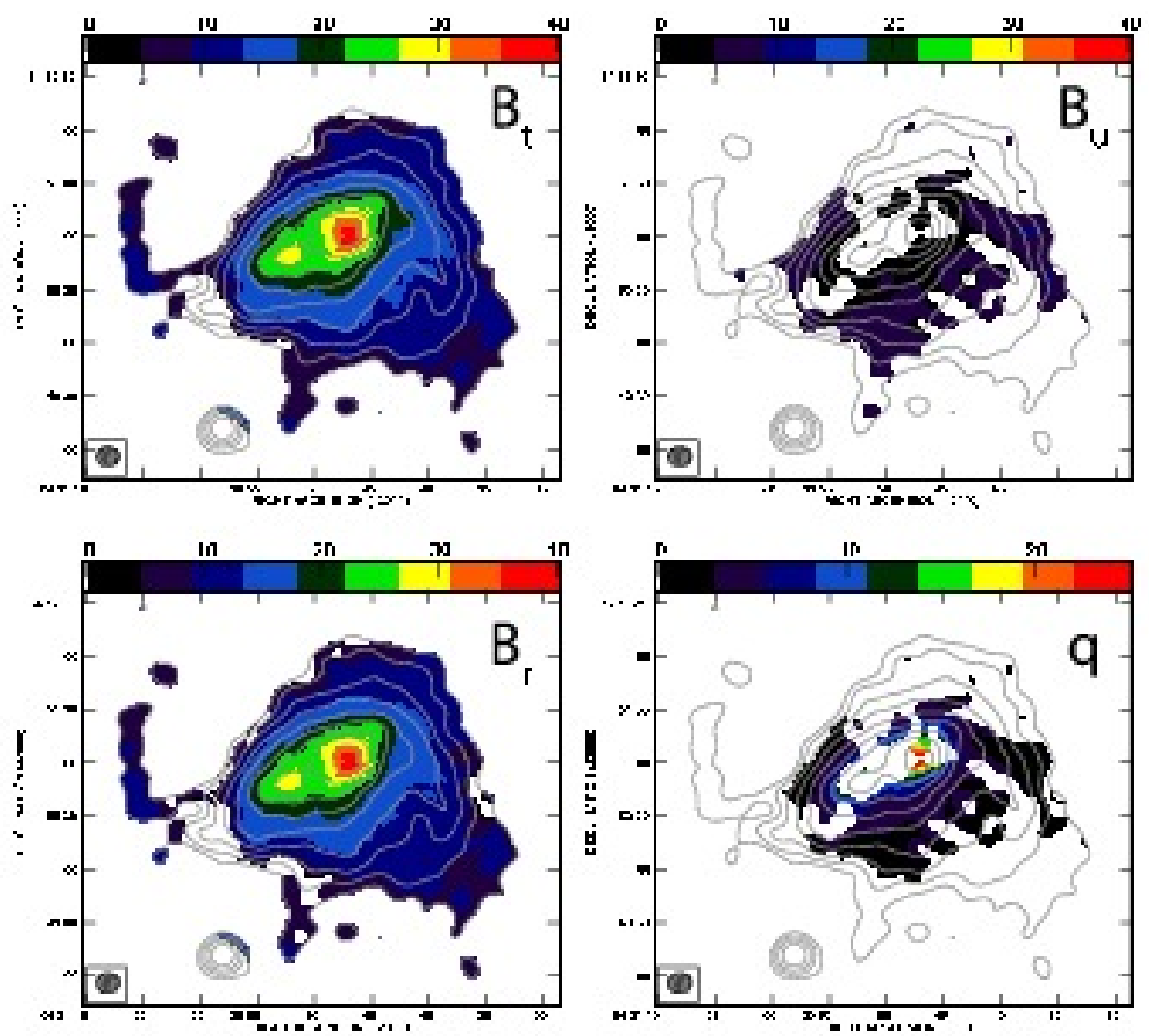

Fig. 9.- Magnetic field strength of NGC 1569 using the $3 \mathrm{~cm}$ data. The color plots are, clockwise from upper left, total magnetic field strength (in $\mu \mathrm{G}$ ), uniform magnetic field strength (in $\mu \mathrm{G}$ ), $q$, which is the ratio of the random magnetic field strength to the uniform magnetic field strength in the plane of the sky (this ratio is usually greater than one since the random field is usually much stronger than the uniform field), and random magnetic field strength (in $\mu \mathrm{G}$ ). See $\$ 4.2$ for the assumptions involved in the derivation of these quantities. The total, uniform, and random magnetic field strength have been corrected for the assumed geometry of NGC 1569 (see Figures 13 and 14. The $3 \mathrm{~cm}$ total intensity emission with 13" resolution is shown by contours. The contours are 3, 6, 12, 24, 48, 96, 192, 384, 768, and 1536 times the $1 \sigma$ noise level. 


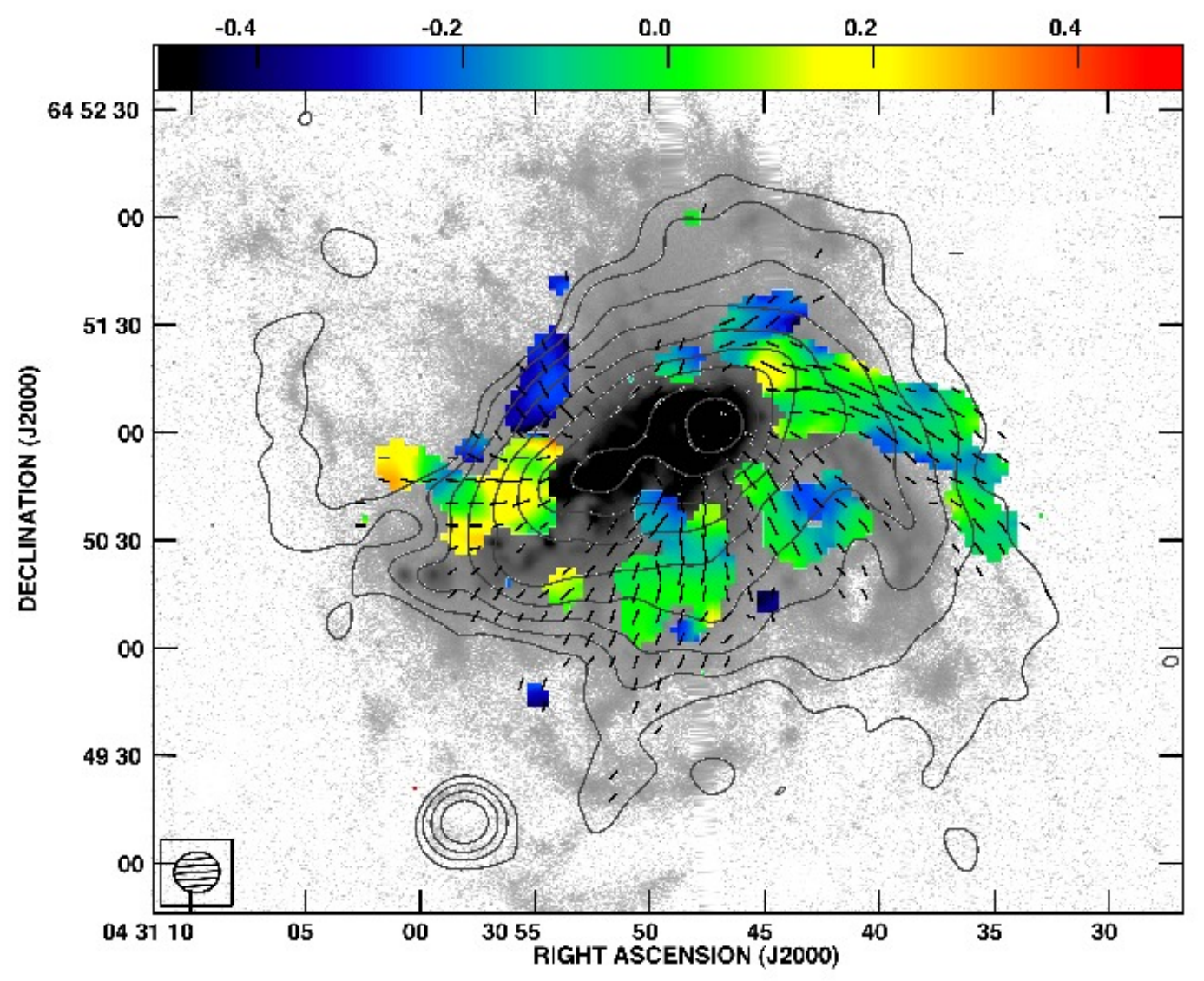

Fig. 10.- Rotation measures between the $14^{\prime \prime}$ resolution $6 \mathrm{~cm}$ and $3 \mathrm{~cm}$ data (color), magnetic field vectors derived from the $3 \mathrm{~cm}$ data, and the contours of the $14^{\prime \prime}$ resolution $3 \mathrm{~cm}$ data overlaid on $\mathrm{H} \alpha$ image courtesy of D. Hunter (Hunter \& Elmegreen 2004). The color bar is in units of $10^{3} \mathrm{rad} \mathrm{m} \mathrm{m}^{-2}$, a $1^{\prime \prime}$ polarization vector has a polarized intensity of $12.8 \mu \mathrm{Jy}_{\text {beam }^{-1}}$, and the contours are 3, 6, 12, 24, 48, 96, 192, 384, 768, and 1536 times the $1 \sigma$ noise. 


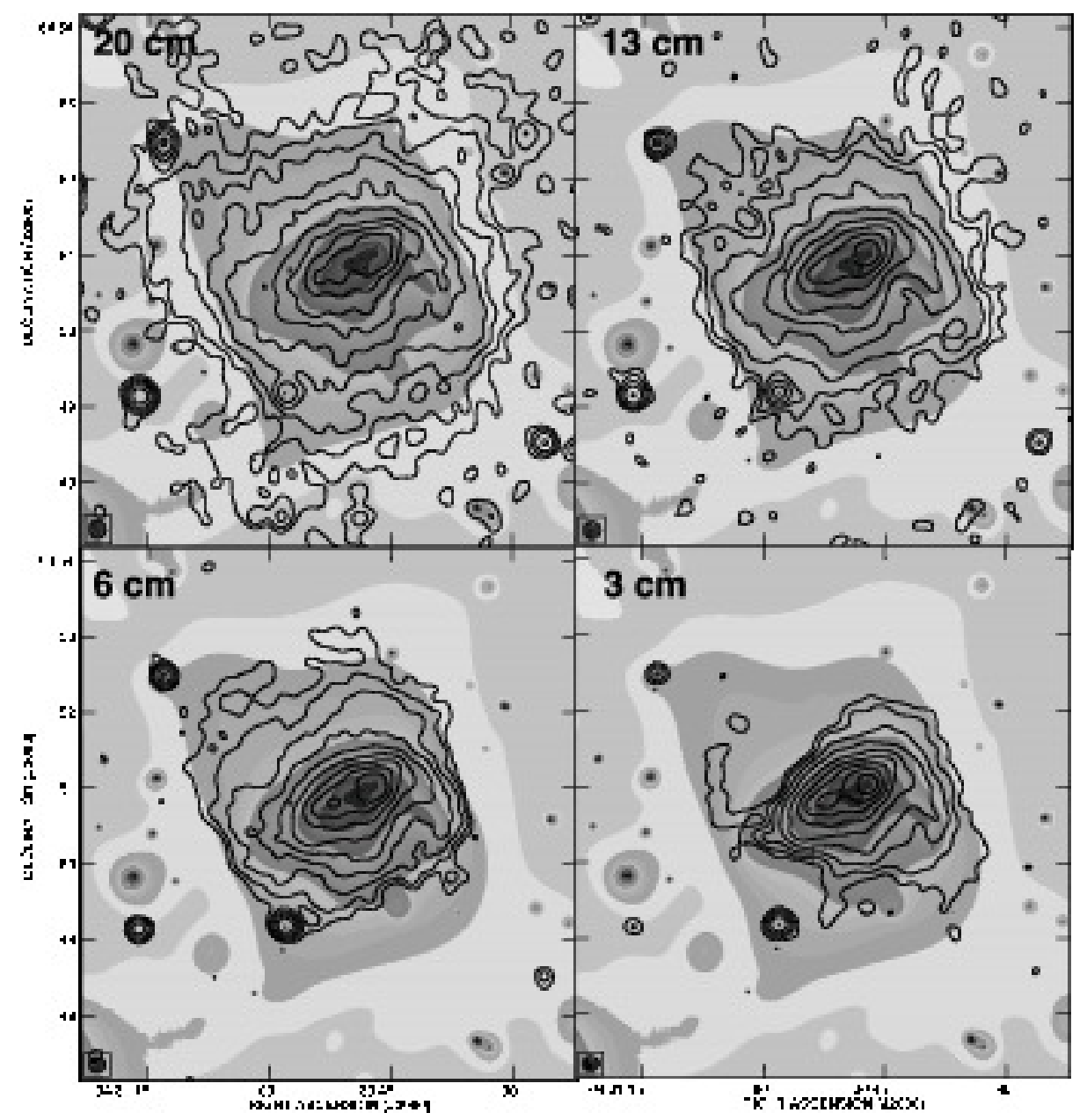

Fig. 11.- Adaptively smoothed, total $(0.3 \mathrm{keV} \leq \mathrm{E} \leq 8.0 \mathrm{keV})$ X-ray emission image from Ott et al. (2005a) with $20 \mathrm{~cm}, 13 \mathrm{~cm}, 6 \mathrm{~cm}$, and $3 \mathrm{~cm}$ contours overlaid. The radio continuum images all have beams with approximately the same size as the $20 \mathrm{~cm}$ beam. The contours start at 3 times the $1 \sigma$ noise level in each band and each subsequent contour increases by a factor of 2. The beam for each wavelength is in the lower left of each panel. The region of dark gray in the lower left hand corner of each panel is not emission from NGC 1569 but instead is due to edge effects from the adaptive smoothing process. 


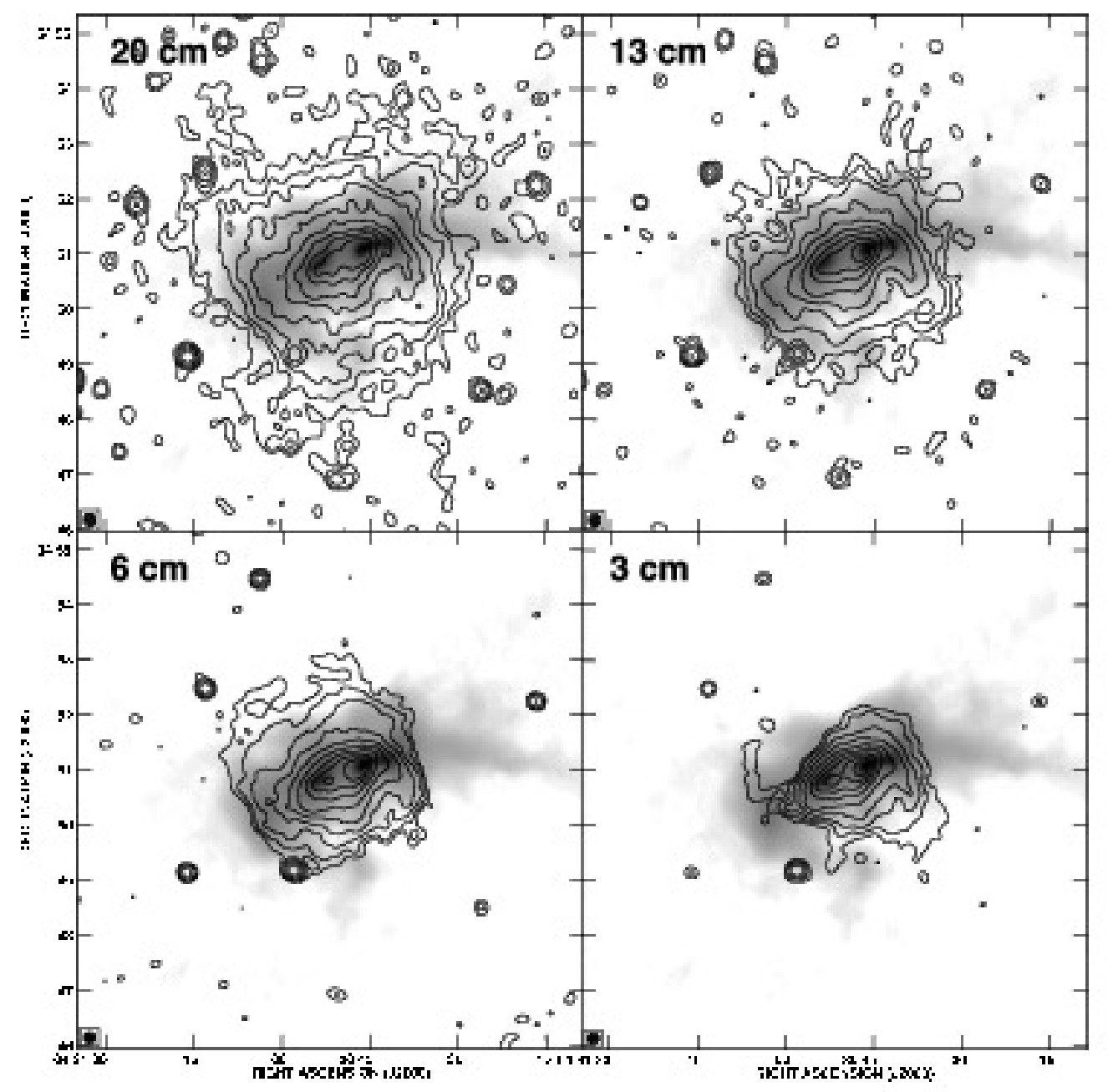

Fig. 12. - Neutral hydrogen emission from Mühle et al. (2005, 2006) with $20 \mathrm{~cm}, 13 \mathrm{~cm}, 6 \mathrm{~cm}$, and $3 \mathrm{~cm}$ contours overlaid. The radio continuum images all have beams with approximately the same size as the $20 \mathrm{~cm}$ beam. The contours start at 3 times the $1 \sigma$ noise level and increase by a factor of two for each subsequent contour. The beam for the radio continuum images is shown the lower left of each panel. 


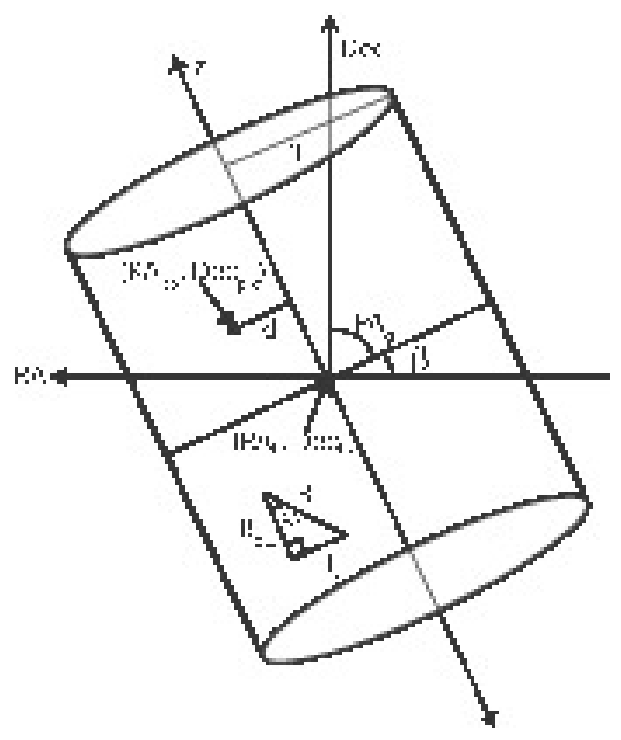

Fig. 13.- Geometry assumed for NGC 1569 in plane of sky.

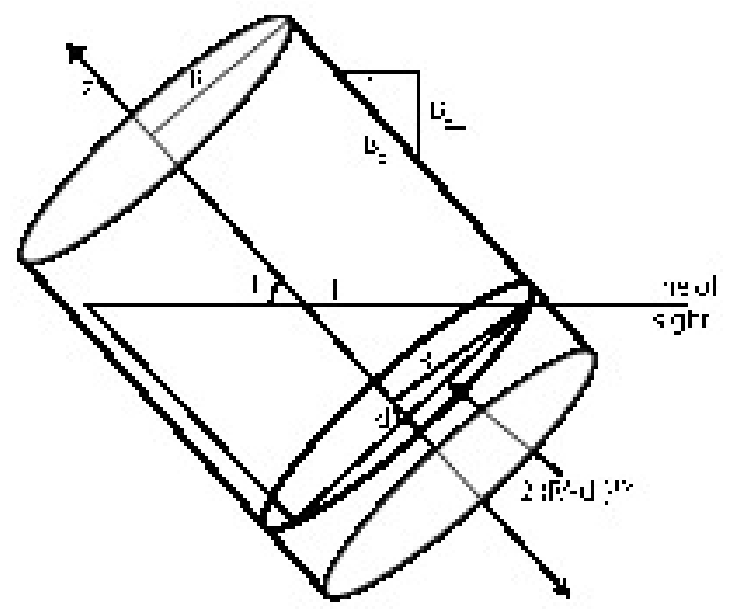

Fig. 14.- Geometry assumed for NGC 1569 along line of sight 